\title{
A survey of Chinese Interpreting Studies: Who influences who ... and why?
}

Ziyun Xu, Leonid B Pekelis

This paper describes how scholars in Chinese Interpreting Studies (CIS) interact with each other and form discrete circles of influence. It also discusses what it means to be an influential scholar in the community and the relationship between an author's choice of research topic and his academic influence. The study examines an all-but-exhaustive collection of 59,303 citations from 1,289 MA theses, 32 doctoral dissertations and 2,909 research papers, combining traditional citation analysis with the newer Social Network Analysis to paint a panorama of CIS. It concludes that the community cannot be broadly divided into Liberal Arts and Empirical Science camps; rather, it comprises several distinct communities with various defining features. The analysis also reveals that the top Western influencers have an array of academic backgrounds and research interests across many different disciplines, whereas their Chinese counterparts are predominantly focused on Interpreting Studies. Last but not least, there is found to be a positive correlation between choosing non-mainstream research topics and having a high level of academic influence in the community. 


\section{A Survey of Chinese Interpreting Studies:}

4

5

6

7

8

9

10

11

12

13

14

15

16

17

18

19

20

21

22

23

24

25

26

27

28

29

\section{Who influences who ... and why?}

\author{
Ziyun $\mathrm{Xu}$ \\ Universitat Rovira i Virgili
}

Leonid Pekelis

Stanford University 


\section{A Survey of Chinese Interpreting Studies:}

Who influences who ... and why?

32 Abstract:

33 This paper describes how scholars in Chinese Interpreting Studies (CIS) interact with each other 34 and form discrete circles of influence. It also discusses what it means to be an influential scholar 35 in the community and the relationship between an author's choice of research topic and his 36 academic influence. The study examines an all-but-exhaustive collection of 59,303 citations 37 from 1,289 MA theses, 32 doctoral dissertations and 2,909 research papers, combining 38 traditional citation analysis with the newer Social Network Analysis to paint a panorama of CIS.

39 It concludes that the community cannot be broadly divided into Liberal Arts and Empirical 40 Science camps; rather, it comprises several distinct communities with various defining features.

41 The analysis also reveals that the top Western influencers have an array of academic 42 backgrounds and research interests across many different disciplines, whereas their Chinese 43 counterparts are predominantly focused on Interpreting Studies. Last but not least, there is found 44 to be a positive correlation between choosing non-mainstream research topics and having a high 45 level of academic influence in the community.

\section{Introduction}

The earliest documentary record of interpreting dates back as far as $3000 \mathrm{BCE}$ - the Ancient Egyptians had a hieroglyph for it (Delisle \& Woodsworth, 1995) - but it can be assumed reasonably safely that the first interpreters started work as soon as cavemen realized they could not be sure to make themselves understood by neighboring tribes using gestures and signs alone.

52 Given its extremely long history, it is somewhat surprising that it only became an independent

53 field of academic enquiry in the 1990s, when scholars began consciously to use the term 54 Interpreting Studies, to distinguish it from the original 'parent' Translation Studies. Despite the 
mystique which still surrounds the profession to a certain degree $^{1}$, since the Second World War interpreters have been increasingly in demand to bridge communicative divides wherever they might arise-war crimes tribunals, peace-keeping operations, high-level international trade negotiations, low-level sightseeing trips ... the list is endless.

Chinese interpreters came to prominence on the international stage when the People's Republic of China (PRC) regained its seat at the United Nations (UN) in 1971. As a result of China's return the UN was instrumental in establishing a dedicated training program to meet the demand for conference interpreting services from various of its offices all over the world (Wang, 2006). The first research article on Chinese Interpreting Studies (CIS) archived by $\mathrm{CNKI}^{2}$ was published in the late 50s (Tang \& Zhou, 1958), and since then the discipline's growth has been explosive: a total of over 3,600 scholars have to date produced nearly 3,000 journal articles and conference proceedings, 1,300 MA theses and over 30 dissertations on the subject. Given its rapid evolution and ever-heightening academic status it is of crucial importance to study the structure of this scientific community. The purpose of the present scientometric survey is to marry the traditional technique of citation analysis with the newer one of Social Network Analysis (SNA) to obtain a fuller picture of the ways in which CIS scholars communicate with each other both formally and informally.

\section{Major questions}

To gain an understanding of how scholars in CIS communicate with one another to generate learning and advance the field, both citation analysis, which describes formal networks of influence (Baumgartner \& Pieters, 2003), and social network analysis, which identifies informal communities (Otte \& Rousseau, 2002), have been used in this study. Using an all-but-exhaustive collection of citation data, we ask how authors interact, how we can characterize who is influential, and what being influential means. These questions have long

\footnotetext{
${ }^{1}$ Until the release in 2005 of the movie The Interpreter, starring Nicole Kidman, many an outsider was no doubt unsure of the difference between written translation and spoken interpreting.

${ }^{2}$ The China National Knowledge Infrastructure is by far the nation's most comprehensive academic database, archiving conference proceedings, journal articles, MA theses and doctoral dissertations dating back to the early 1900s.
} 
81 attracted attention in the scientific community (see for example Van Dalen \& Henkens, 2001; 82 Haslam et al., 2008; Buela-Casal et al., 2009; Chen \& Redner, 2010; Ravallion \& Wagstaff, 83 2011), partly in consequence of Kuhn's (1970) seminal work on the nature of science, in which 84 he emphasizes the importance of adopting a data-driven approach to analyzing the structure of 85 the scientific community, and partly because academic authorities are ever vigilant to ensure that 86 the investments they make in their researchers' projects are justified by the results - that they are, in short, spending their money wisely. We also aim to discover which locations and institutions carry the most weight in terms of academic influence, and how their impact changes over time. These questions merit attention because a location's economic status often plays an important role in determining the level of funding it devotes to research and because, as (Gile, 2013a) points out, institutions are the driving force behind most CIS research.

\section{3. Literature Review}

93

The growth of scientometrics in China has trailed developments in the West by about a decade. In an article in People's Daily in 1977, Hsue-Shen Tsien, a scientist influential in the development of missiles and space programs in both China and the United States, argued for the need to establish an independent discipline that focused on the 'science of science'. Hongzhou Zhao is considered the pioneer of scientometric research in China. While working at a labor camp in Henan Province in 1974 he analyzed the History of Natural Science published by the Fudan University Journal, studying the way in which research production centers 'shift' from place to place around the world over time (Liu, 1999). In 1985 he and Gouhua Jiang published in Scientometrics an article on the demographics of scientists - this was the first time a Chineseauthored article on the subject received widespread recognition from the international community (Zhao and Jiang, 1985). In 1978 the Research Group for the Science of Science became the first academic body in China to study scientometrics (Jiang, 2008).

China developed its own citation indexes, though they appeared much later than in the West and are still not fully comprehensive or standardized. The leading ones include the state-funded Chinese Science Citation Database (CSCD), created in 1989, and the Chinese Social Sciences Citation Index (CSSCI), established in 1998. Unlike its Western counterpart the Social Sciences 
111 Citation Index (SSCI), the CSSCI collects data from monographs, collective volumes and

112 miscellaneous websites in addition to journal articles. However, while the SSCI includes articles

113 dating back to 1972 , the Chinese equivalent contains those published only since 1998, seriously

114 limiting the pool that researchers can draw on for analysis. As an example, a search for

115 'Interpreting' in the CSSCI currently yields only 263 entries; by contrast, the same search of

116 SSCI produces 585.

118 Despite the difficulty in accessing data, multiple scientometric studies across various academic 119 disciplines have been carried out in China (see for example, Zhang \& Zhang, 1997; Wang et al., 120 2005; Ruan, 2012). A handful of researchers have applied the principles and methods of 121 scientometrics to CIS. For example, some have attempted to provide a broad overview of trends 122 and developments in the discipline by classifying relevant journal articles by theme and giving a 123 few examples of the leading articles in each category (Hu \& Sheng, 2000; Liu \& Wang, 2007; $124 \mathrm{Li}, 2007)$. Others have gone further by backing up their claims with simple counts of articles 125 published on a given theme (Mu \& Wang, 2009; Tang, 2010).

127 Gao Bin (2008) and Zhang Wei (2011) have published studies of the similarities and 128 differences between translation and interpreting research in China and in the West. Whereas 129 Zhang takes a broad view, covering the entire subject of interpreting, Gao's interest is in the 130 cognition aspect of Simultaneous Interpreting Studies ${ }^{3}$. The latter's corpus of articles includes 131 ones from eight leading Western academic journals ${ }^{4}$ published between 2000 and 2007, and ones 132 published in three premier Chinese journals ${ }^{5}$ between 1994 and 2007. Her analysis suggested that 133 recent works in the West showed fewer signs of debate over the nature of interpreting than were 134 common previously, and that instead they frequently drew on findings from contemporary 135 psychology to re-evaluate prior research in the field. Gao noted the strong influence on CIS of 136 late-20th-century major Western theories such as Gile's Effort Model, cognitive pragmatics and 137 the Interpretive Theory, and observed that, unlike their colleagues in the West, Chinese

\footnotetext{
3 This sub-discipline deals with the various aspects of how interpreters manage to render a speaker of one language's meaning into another at the same time as they are speaking.

${ }^{4}$ The Western journals are: Interpreters' Newsletter, Interpreting, Meta, The Translator, Babel, Hermes, Target and Forum.

${ }^{5}$ The Chinese journals are: Chinese Translators Journal, Chinese Science Technology Translators' Journal and Shanghai Journal of Translators for Science and Technology.
} 
138 researchers were less inclined to interdisciplinarity.

\section{The Present Study}

141

142 There is clearly a growing interest in scientometrics among CIS researchers. Aside from Gao, 143 most authors to date have applied its more basic methods and principles, mainly article counts, in 144 their research. This is the logical place to start, but there is ample room to employ the more 145 complex approaches scientometrics offers. Doing so will shed light on the true impact made by 146 individual scholars, and will provide other more finely tuned information about the evolution of 147 specific fields of research in a relatively objective way. As (Lowry et al., 2007) point out, mere 148 numbers do not permit a nuanced analysis of influences within a given discipline; for example, 149 an author may have published numerous articles but have little influence among his peers. In

150 addition, a small sample population may cause significant biases and affect the outcome of any 151 analysis conducted. The present research study is intended to contribute to CIS by carrying out, 152 for perhaps the first time, a thorough scientometric survey of the literature, including journal 153 articles, theses and dissertations. Its aim is to provide scholars with a comprehensive and 154 objective overview of the interactions between scholars in the field, and of which academics are 155 the most influential and how their choice of particular subjects of enquiry relates to their impact 156 on research as a whole.

\section{$157 \quad$ 4.1 Research Questions}

159 Expanding on the major questions outlined at the beginning of this paper, three specific research questions were developed to ascertain how CIS scholars interact each other and how

161 influence is defined in the community. The following section outlines the author's rationale for 162 investigating each research question. so, what are the features of those communities? 
166 The advancement of a science relies heavily on its participants' communicating and 167 collaborating with one another: scholars build on each other's research, and work together to 168 address common issues or to replicate colleagues' experiments under different conditions to 169 investigate whether their conclusions can be extrapolated to a larger population. In the context of 170 citation analysis, identifying community structures can help us understand the predominant 171 research themes in a given field and how certain subject matters grow or decline in popularity 172 over time.

173

174 2. In terms of citations, who are the most influential scholars in the CIS literature?

175 Nederhof (2006) observed significant differences in citation behavior between natural and 176 social sciences: members of the former communities (physics, chemistry, etc.) tended to 177 influence each other across geographical boundaries, whereas those of the latter (sociology, 178 linguistics, etc.) generally had very limited influence beyond the countries in which they lived. 179 One might think Interpreting Studies would be an exception to this rule: because of its focus on 180 the interactions between languages and cultures, its authors might reasonably be expected to 181 exert influence across the boundaries of language, geography and culture. Identifying the most 182 influential scholars in CIS ought to reveal whether any Western researchers have an impact on 183 the Chinese field and if so why; it should also prove useful for identifying differences between 184 the most influential Western and Chinese authors' backgrounds, and for exploring the dominant 185 schools of thought in CIS.

186

187 3. Are there any research topics that influential scholars tend to write about? What themes 188 and keywords correlate with author influence?

189 All researchers would like to see their papers frequently cited by others, and hope that their 190 colleagues might be inspired to pursue their work and address any questions that may remain 191 unanswered. However, the reality is that few articles published are highly influential: a far higher 192 number are rarely read or cited by others. Studying what makes an article influential is a useful 193 exercise from three perspectives: firstly, every scholar would like to make a mark within his 194 research community, and so would do well to know what makes for a successful paper; secondly, 195 because the bodies which allocate grants and other forms of funding always want to be assured 196 that their investments are money well spent (generally speaking, scholars need to publish when 
197 they receive a grant); and lastly, studying these predictors of influence can help to identify the 198 hottest topics in the field. A handful of researchers have already explored the issue of what makes one article influential and another not. Buela-Casal et al. (2009) examined the relative

200 influence of theoretical and empirical papers in three Spanish psychology journals, concluding 201 that the former type received twice as many citations as the latter. Haslam et al. (2008) studied 202 the citation data for 308 articles in social-personality psychology, and found the following 203 factors particularly strong for predicting an article's impact: (1) the reputation of the first-listed 204 author; (2) the presence of a senior colleague's name among a new author's collaborators; and 205 (3) a journal's ranking. The present study is the first time that the predictors of influence in CIS 206 have been analyzed.

\subsection{Data Collection and Organization}

Given the paucity of coverage of CIS citation data in existing academic databases, for the

211 present study a near-comprehensive database of 59,303 citations was built from scratch - they

212 represent citations from the 1,289 Chinese MA theses, 32 doctoral dissertations and 2,909

213 research papers available to the author. These three bodies of literature, chosen because they best

214 represent the overall state of CIS, were accessed through multiple channels: field trips to

215 university libraries, interlibrary loans, book purchases, and academic databases such as CNKI,

216 Wanfang and the National Digital Library of Theses and Dissertations in Taiwan - thus ensuring 217 wide coverage of academic works in both mainland China and Taiwan. Once collected, the 218 references were manually entered into Excel Spreadsheets, using the idea behind Structured 219 Query Language (SQL) to managing data. The method employs multiple interactive and cross220 referenced tables; in the present case there are three such: Documents, Authors, and Citations. 221 For these tables to interact with one another it is important to have unique 'keys', elements that 222 allow each row to be identified: a unique, consistent 'author key', for example, enables us to 223 know whether a particular person in the Authors table is the same as one found in a row of the 224 Documents table. 
Figure 1: Data organization framework using the idea behind SQL relational data management system. Arrows between tables show examples of unique pieces of information which are indexed in multiple tables, as a way of linking data points between tables.

231 The key concept behind the present analysis is that of the citation network: the documents are nodes in this network, with arrows interconnecting them when authors cite their predecessors.

233 The nodes are labeled with various attributes such as 'author', 'publication year', and 234 'keywords'. The number of nodes associated with an author is the number of documents he has produced. Authors share a node when they have co-authored a document. Arrows are in the direction of the citation, so the cited work is at the receiving end of the arrow. When arrows are used to indicate the presence of a citation between documents, there can be at most one arrow between each pair of nodes; when they are used to indicate a type of citation, there can be multiple arrows, as one document may cite another multiple times. A total of ten Excel Spreadsheets were compiled: three documents table for CIS doctoral dissertations, MA theses and academic papers; six citation tables - CIS doctoral dissertations (English and Chinese citations), MA theses (English and Chinese citations), and academic papers (English and Chinese citations), and one author table. This data format makes it easy to be exported into Tulip for data visualization (see section 5.1) and ' $R$ ' for correlational analysis (see section 5.3). The raw data alongside the source codes has been deposited into GitHub, and can be accessed from this link: https://github.com/danielxu85/CIS

\subsection{Description of Topic Labeling Method}

Rather than rely solely on the keywords provided by the papers' authors, the content of each and every publication in the data-set was carefully analyzed with the aim of generating keywords that best captured the topics they covered. The keywords typically chosen by authors can often were further grouped into six broad themes: Training, Professional, Language, Socio-cultural, between categories. It should be noted, though, that each paper under examination may be tagged 
258 with multiple themes depending on its content. Consolidating keywords into themed categories 259 can help identify major trends in CIS which might otherwise have been drowned out by the sheer 260 number of keywords generated.

\section{5. Methodology}

\subsection{Author interaction}

263

264 Using near-exhaustive citation data, the present authors wished to determine whether the 265 communities of CIS are best classified according to the hypotheses put forward by earlier scholars (Moser-Mercer, 1994; Gile, 2005 \& 2013b). In the first two of these studies it was noted that there existed two dominant and opposing camps - those who approached research from a liberal arts standpoint and those who leaned towards natural/empirical science - and that there was little communication between the two. In his later study Gile refined his original classification by suggesting that Translation \& Interpreting Studies can be divided into three broad groupings-Human Sciences Culture, Canonical Scientific Culture, and Practice,

272 Reflection, Generalization.

273

To address this aspect of CIS, visual representations of its citation networks were created. A 275 number of open source software applications are available for analyzing and visualizing networks: some, such as SciMAT and VantagePoint, are well suited for analysis, while others (e.g. Pajek and Gephi) are better for visualization. Tulip, a program designed for analyzing and visualizing relational data, provides a good balance because it incorporates a number of layout and clustering algorithms, in addition to network metrics. More importantly, Tulip was selected over other applications for its capacity to visualize large complex networks - the complete citation network used in the present study contains over 12,000 nodes and in excess of 50,000 282 edges.

284 Tulip enabled us to import the entire CIS citation network data in the form of a list of all edges 285 (connections between nodes) in the data set. Once this was done it gave the option to apply a 
286

287

288

289

290

291

292

293

294

295

296

297

298

299

300

301

302

303

304

305

306

307

308

309

310

311

312

313 PageRanks were calculated using the Algorithm-Measure-Graph-PageRank option in Tulip.

314 The top-ranking 150 nodes were selected, and an induced sub-graph ${ }^{6}$ was created which showed

\footnotetext{
${ }^{6}$ An induced sub-graph is one that highlights only a certain number of nodes and all the edges connecting them, but omits all the other nodes and non-connecting edges.
}

number of layout algorithms. In the present instance, once the entire CIS citation network had been imported, we selected a force-directed algorithm-Fast Multipole Multilevel Method (FM ${ }^{3}$ ) - to lay out the graphs presented in the images seen in Figures $3 \& 4$ (Hachul \& Jünger, 2005). This algorithm groups nodes that are multiply connected to each other closer together in the layout while distancing ones that are not directly connected. This approach makes the produced images ideally suited for visually detecting community structures in networks.

A panoramic graph of the research interactions between various CIS authors was created using the 2012 citation data. In this graph vertices represent authors and the edges represent the number of citations between them. To generate a full network image with Tulip software, a placement algorithm was used to lay out the nodes. The edges were rendered invisible to ensure that the nodes could be seen clearly. The node color was set to blue using the property option available in Tulip.

To study the relationships between the most influential nodes in the network, we firstly filtered out the less important ones, retaining only the top 150, then calculated the PageRank score of each that remained. The PageRank algorithm for citation networks measures the importance of an author by gauging the quality and quantity of other authors that cite him. The author whose PageRank is being calculated ('the target') is said to be at a distance of 0 from himself, and each author who cites him at a distance of 1 . The importance of these Distance 1 authors is in turn calculated by the quality and quantity of authors citing them, who are at a distance of 2 from the target. The underlying idea is that influential authors will be much cited by other influential authors, while non-influential authors will either not be cited or only be cited by other noninfluential authors. The process continues to a pre-determined parameter known as the damping factor. In line with the majority of citation studies, which use an average chain length of 10 to 15 , for the present study a damping factor of 0.15 was used. 
315 only these 150 . Again, the $\mathrm{FM}^{\wedge} 3$ algorithm was used to lay out the nodes, and Edge Bundling 316 was used to merge edges which were close to one another to make the layout more readable.

317 Tulip automatically colors nodes as soon as any metric has been calculated. In the present

318 instance the nodes were colored using a gradient scale from blue (high) to orange (low) values.

319 Since it was not possible to visualize the names of all the authors, we chose to display only nodes

320 with a high value of any metric, which in this case was PageRank.

321

322 In addition to visually identifying the communities within CIS, a quantitative analysis was 323 conducted to verify whether the network had two or more clusters. Community detection 324 pinpoints the most natural groupings of individuals present in a network (Schaeffer, 2007). There 325 are a number of ways to evaluate the quality of such groupings, one of the most widely used in 326 recent times being Q Modularity, which was introduced by Newman in 2004.

327

328 Modularity is defined as the percentage of all connections that fall within a community, minus 329 the expected percentage of connections in the community. The expected percentage is based on 330 the assumption that connections are distributed completely at random, with no regard for 331 community structure. The modularity cut algorithm determines how to partition a graph into 332 communities with high modularity scores, hence the communities it identifies all contain a 333 higher number of intra-community connections than might be expected to occur purely at 334 random. Modularity values lie between 0 and 1; higher values are desirable and represent better 335 clusterings. Typical values lie in the range 0.3 to 0.7 (Newman, 2004), values between 0.0 and 3360.2 suggesting that the graph is entirely random with no known community structures (Rényi \& 337 Erdős, 1959).

338

339 Seven commonly used network clustering algorithms were used to determine the number of 340 clusters and optimize the process of clustering CIS citation data; Spin-glass stood out as the best 341 at defining CIS communities. Modularity values, which were obtained for different numbers of 342 clusters using the Spin-glass algorithm, indicate the optimum number of clusters for CIS.

344 Finally, to analyze the common features of the discrete communities of CIS, the image of 345 clusters was generated. The Spin-glass algorithm was applied with seven clusters. Subgraphs of 
346 each cluster were generated with a unique color for each different cluster. $\mathrm{FM}^{\wedge} 3$ was used to

347 layout the nodes of each cluster individually by executing the layout algorithm on each of the

348 subgraphs. Again, the edges were rendered invisible to make the graphs more readable, and

349 nodes were labeled with author names. PageRank was employed to identify important nodes and

350 labels were displayed only for the most important from each cluster.

351

352 It should be emphasized here that modularity cut does not involve the use of meta-information

353 about authors to determine how they are divided into communities. Previous researchers in SNA

354 have not attempted to use meta-data about each citation to generalize the features of each

355 community, because a variety of factors can drive authors into a certain community: a

356 connection can be established between two authors because they have co-authored an article

357 together, or because they have cited one another's research, a process known as co-author

358 citation (Newman, 2001). In addition, meta-information, such as the content of each cited paper

359 and background information regarding each cited author, cannot be obtained from commercial

360 databases, which means manual labor is required to obtain and screen thousands of papers.

361 Newman and Girvan (2004) applied the modularity cut algorithm to identify communities based

362 on co-authorship data: authors were added to the network as vertices, edges between them

363 indicating their co-authorship of one or more papers in the data-set. Newman and Girvan were

364 primarily interested in investigating whether researchers from the same community were

365 acquainted with one another. Takeda and Kajikawa (2010) analyzed citation data in the fields of

366 energy and material science by tracking modularity scores obtained from each clustering

367 iteration, but they stopped short at summarizing the features that define each community:

368 clustering only indicates that the nodes in each group are similar, but the similarity is dependent

369 upon whether the nodes are connected, not why they are connected.

370

\section{$371 \quad 5.2$ Most influential scholars}

372

373 All the Western and Chinese authors appearing in the citation data were ranked according to 374 their degree centrality (DC) ${ }^{7}$ and weighted degree centrality (WDC) ${ }^{8}$ measures, and to their

\footnotetext{
${ }^{7}$ Degree centrality calculates the number of edges connected to a particular node in the network. It has two sub-
} 
375 PageRank Algorithm (PRA) scores. DC and WDC are first-order centrality measures, while PRA 376 is a higher-order measure. The three highlight different aspects of an author's influence within a 377 network: for example, out-degree and weighted out-degree measures indicate how well authors 378 disseminate information about the work of other scholars, while PRA assigns them scores based 379 on how well connected they are with influential colleagues.

380

381 It was expected that some authors, despite publishing prolifically, would be found to have little 382 research impact within the CIS community, while others, despite publishing very little, may 383 nonetheless be widely cited. There were also grounds to expect that the ways in which 384 influential authors are cited may be subject to variation: they might be highly influential thanks 385 to a wide range of much-cited work, or their influence might depend on a small number of seminal works. In addition, the present author ventured to predict that, despite the homogenous background of CIS researchers (Zhang 2008), authors from other disciplines may have topped the influence polls.

\subsection{Research topics and academic influence}

There are various methods for measuring an author's influence, such as DC, WDC and PRA

393 (see Question 2 above), but for this analysis only in-degree centrality, out-degree centrality, PRA and EigenVector centrality were used. WDC was excluded because it and DC are both first-order centrality measures: they essentially measure the same aspect of an author's influence. The present study's database contains 2,909 research articles, 1,289 MA theses and 32 doctoral dissertations; from these were extracted 978 unique keywords to describe their contents. All the keywords for each author in the database were tallied up, and a keyword profile created for each, representing the relative frequency with which he or she used a keyword, normalized to 1, i.e. the number of times that keyword was used divided by the total number of keywords he or she used-hence a keyword with a value of 0.3 represents $30 \%$ of all keywords used by that author.

categories: an author's in-degree centrality represents the number of other authors citing him, while his out-degree is the number of others cited by him.

8 Weighted degree centrality is the total number of citations an author makes and receives. Weighted in-degree centrality is the total number of citations of his work made by others, while weighted out-degree centrality is the total number of citations he makes in all his publications. 
402 Normalization was added in to prevent there being undue emphasis on the connection between 403 keywords and centrality measures when identifying prolific authors.

404

Such a large number of keywords has two limitations. Firstly, many of them are synonyms or 406 hypernyms, leading to conceptual overlap. Second, in any regression the larger the number of 408 explanatory variables, the more data they require in order to maintain statistical power- the ability to detect significant relationships between explanatory variables and the response (see for example Hsieh, Bloch, \& Larsen, 1998). In the present case the explanatory variables, namely the relative frequencies of the almost 1,000 keywords, were too numerous in relation to the number of documents for the 'bulk' statistics to yield good results. To avoid this sizable stumbling-block the keywords were classified into six themed groups: Cognitive, Language, Professional, Socio-cultural, Training and Miscellaneous. The categories were designed to be non-overlapping so as to allow for the drawing-out of meaningful trends which would otherwise be undetectable amid the crowd of keywords present in the documents. A supplementary analysis 417 section.

419 The authors' network measures (in-degree centrality, out-degree centrality, PRA and 420 EigenVector centrality) were first matched to their theme profiles, which came from separate databases: journal articles, MA theses, and PhD dissertations. Of the 2,277 journal article authors, 1,023 were matched in the network database; of the 1,289 MA theses authors, 1,092 were matched; and finally, of the $32 \mathrm{PhD}$ authors, 29 were matched. In total, roughly $60 \%$ of authors in the theme profiles database were matched with network measures ${ }^{9}$. Following the same methodology, a subsequent mapping of keywords to themes was also performed.

The reason that $40 \%$ of the authors could not be matched in the network influence database was 428 that no references were available in their works. The majority of these authors did not include 429 any bibliography in their papers, and the works of a very small proportion-mainly authors of theses - are embargoed by their affiliated institutions. This is an inherent property of the data, 431 and it is an important component to document when describing the customs of different 
432 academic cultures. Because of China's unique intellectual traditions in the early stages of CIS' 433 development as an academic discipline, the overwhelming majority of papers published had no 434 bibliographic references. In addition, many early papers were case studies of the authors' own 435 experiences, and their documentary nature precluded the need for many citations. However, 436 these early studies were included in the data-set for three reasons. Firstly, these articles were 437 produced during CIS's initial stage, as per Schneider's model of the development of scientific 438 disciplines (2009), and to exclude them would be to miss out on a significant portion of the early 439 literature. Secondly, many of them received citations from later studies, which indicated that they 440 served as the foundation for the development of CIS and brought academic value to the field.

To investigate how well themes act as predictors of the network influences of CIS authors, the most simple approach was linear regression; this is a good starting point for becoming 444 acquainted with the data and is typically used as a first step in statistical analysis for examining the null hypothesis that the explanatory variables have no relationship at all to the response variable. For the preliminary analysis, separate linear regression models were fit with theme profiles as the predictors, using DC, WDC, PRA and EigenVector as a response variable in each model. Since each theme profile was normalized, authors who published numerous papers and those who published only a few had similar-looking theme profiles, therefore the number of papers published by an author was added as a predictor. An F-test was used to determine if any of the regression models could explain variation in author influence in statistically significant terms. The F-test results showed that the linear regressions did not explain the variations in author influence very well. High levels of disparity between authors' influences was the suspected reason for this. To confirm this suspicion, the disparity was calculated by means of the Gini Coefficient, which measures the concentration of mass in a cumulative distribution, and is borrowed from the field of economics.

A linear model is a simple approach but it makes strong assumptions about the relationship 459 between the response variable and the explanatory variables, therefore it came as no surprise that 460 the linear regression failed to explain the data well. The next approach adopted was one which, though less ambitious in terms of explanatory power, is far less dependent on assumptions. This alternative approach involved dividing network measures into three groups ('bins'): high-, 
463 middle-, and low-ranking. However, rather than assuming that these three bins were equally 464 probable and so spacing their cutoffs regularly, a data-based approach was employed to 465 determine where they should fall. A total of 20 cutoff points were considered, corresponding to 466 the percentiles of network measures, from 0 to 95 in steps of 5. Creating the three groups called 467 for finding specific lower and upper cutoffs for the middle-ranking group. An example of where 468 the cutoffs between the three bins might be placed would be at the 5th percentile between the 469 low- and middle-ranking groups, and at the 85th between middle- and high-ranking (see Figure 470 2).

471

472

473 Figure 2: Ranking group cutoffs. $5 \%$ of all authors are in the low-ranking group, $15 \%$ in the high-ranking, and the 474 remaining $80 \%$ in the middle-ranking.

The effect of an author's theme profile on the probability of his or her belonging to each of the three groups was estimated by means of a multinomial regression. For each of the different groups a statistical analysis known as a deviance test was performed to assess whether its divisions were good for explaining authors' influence based on their theme profiles and number of publications. This procedure allows a numerical quantity known as the $p$-value to be 482 calculated. For each model the smaller the $p$-value, the more likely the model is a good fit.

Given that there were 190 different combinations of cutoff points to consider for each response 485 variable, we could not simply report as significant those cutoff points that had a $p$-value of less than 0.05 . If we did, by the definition of a $p$-value, we could expect about 10 cutoff points to be 486 significant even if there was no relationship between authors' meme profiles and influence for any cutoff point. Therefore a statistical procedure was used to find cutoff points that gave considerable evidence of a good model fit over and above the fact that we were choosing from 190 different models. This procedure can be measured by a quantity called False Discovery Rate (FDR), which is defined as the expected proportion of false discoveries, or cutoff points that are not significantly related to authors' theme profiles, from all cutoff points detected as significant.

492 In statistics the FDR can be controlled by using the Benjamini-Hochberg procedure. The smaller 493 a group of models' FDR, the greater their chance of representing true underlying effects rather 
494 than random variation in the data. The procedure assigns to each model a score called the $q$ 495 value. One way of interpreting this value is that to build a group of models with a certain given 496 maximum FDR, only those models with a $q$-value below that FDR should be included. 497 Consequently a model is considered good enough for inclusion in a group if it has a small $q$ 498 value.

499

500 Several hypotheses were formulated before the statistical analysis was conducted. One such 501 was that scholars typically perform literature searches by submitting keywords to search engines 502 that rank results from the most recent to the oldest. Under this hypothesis it was expected that 503 authors writing on commonly studied subjects would, because of sheer weight of numbers, have 504 difficulty becoming highly influential. Conversely, authors writing about rarely-studied subject 505 matters would be far more likely to receive attention from colleagues tackling the same subjects, 506 translating into numerous incoming citations for them. Another reasonable hypothesis was that 507 authors might use other methods of performing literature searches, such as finding citations in 508 existing papers or sorting results based on relevance rather than how recently the items were 509 published. The analytical methodology described in this paragraphs is an important first step towards testing the veracity or otherwise of these hypothetical scenarios.

512 To examine whether CIS authors' full keyword profiles were significantly correlated with any 513 of the network measures, one additional analysis was performed-regularized regression. The 514 assumption made at this point was that the majority of keywords were not highly correlated with 515 influence, while a small minority were. In statistics this is called a sparsity assumption (Hurley \& 516 Rickard, 2009). Had a simple linear regression of measure of influence been run on keyword 517 profiles, we would have expected to obtain a large number of very small regression coefficients 518 (one for each unique keyword), some medium-sized, and maybe a few large ones. Adding too 519 many non-significant terms into a standard regression would have obscured the signal from 520 significant terms, hence the need to use regularized regression for removing non-significant 521 keywords. Regularized regression addressed this issue by zeroing out many of the insignificant 522 coefficients. More specifically, a regularization technique called Lasso was run for multinomial 523 regression (Tibshirani 1996) with 10-fold cross-validation to approximate the optimal set of non524 significant keywords and set their coefficients to 0 . The remaining keywords were considered to 
525 be significantly correlated with the network measure. Similar outcomes were hypothesized from 526 the keyword profile analysis as from the earlier theme analysis. Some of the frequently used 527 keywords were expected to be correlated with the low influence group, whereas some rarely used 528 ones were expected to be correlated with high influence. The reason for this predicted outcome 529 was the same as the one for themes described in the previous paragraph: authors whose papers 530 have unique keywords are more likely to be read and cited by fellow researchers than those with 531 common keywords.

\section{6. Results and discussions}

533

534

535

536

537

538

539

540

541

542

543

544

545

546

547

548

549

550

551

552

553

\subsection{Author interaction}

The nodes representing authors were situated in Figure 3 using $\mathrm{FM}^{3}$. Contrary to the expectation that the field of CIS is composed of polarized camps which barely communicate with one another, Figure 3 suggests rather that its scholars cannot be easily divided into clearly separable communities. In addition, the degree distribution of the entire CIS graph follows a scale-free behavior, which implies that several nodes with high In-Degree and Out-Degree scores perform the function of holding the graph together: these nodes are to be found at the center of Figure 3, those with lower In-Degree and Out-Degree scores being pushed towards the periphery. To corroborate this finding, the citation patterns of the top 200 CIS authors were also visualized (see Figure 4). The ranking of these authors was determined using the PageRank algorithm on the entire network. If there were to be well-defined communities, they would have been clearly visible, but in reality Figure 4 also illustrated a hairball effect, which means that even the top-ranking authors closely cite each other's works.

This finding suggests that CIS researchers do not form opposing camps marked by a distinct intellectual preference for liberal arts or empirical sciences, and that certain influential scholars are well-cited across the board. This is in sharp contrast to the situation in WIS, where for personal and professional reasons authors may choose not to include a certain items of relevant research in their works: scholars from ESIT, France's most famous IS institute, almost exclusively cite one another's work and avoid research by scholars from disciplines outside 
554 interpreting; the latter reciprocate by ignoring the interpreting specialists' work, adjudging it to 555 be 'unscientific' (Gile, 1999).

Figure 3: Entire CIS network laid out using a force-directed algorithm

Figure 4: Studying the behavior of interconnections of the top 150 authors. Nodes are placed using a circular algorithm where the most important nodes are displayed in the center to highlight top ranked authors.

To investigate CIS community structures in greater depth a number of leading networkclustering algorithms were examined; the purpose of these was to optimize the clustering process and determine the optimum number of clusters. Table 1 shows the modularity values that resulted from testing each algorithm using CIS citation data to cluster the network. Spin-glass yielded the highest modularity value with seven clusters, so was adopted for this study (Fortunato, 2010). Another reason for using this algorithm is that it allows the user to input the number of clusters required by specifying the number of spins in the system. Other clustering algorithms do not generally allow the number of clusters to be used as input as they determine the number of clusters by optimizing some objective function or by optimizing some dynamic process.

Table 1: Comparison of different clustering algorithms using CIS data

The Spin-glass clustering algorithm is based on spin models, which are popular in statistical mechanics (Reichardt \& Bornholdt, 2004). The underlying idea is based on the principle that nodes connected to each other should belong to the same cluster and ones not directly connected should belong to different clusters. If Potts spin variables are assigned to the vertices of a network, and if the interactions are between neighboring spins, structural clusters can be found from spin alignment of the system. Spins of nodes within clusters are similar and different across 584 clusters with the purpose of maximizing Potts energy. 
587 Figure 5 displays the seven clusters obtained when the Spin-glass clustering algorithm was 588 applied to the CIS citation data network. Nodes in each of these clusters were assigned their 589 positions using the aforementioned $\mathrm{FM}^{3}$ algorithm. Their closely-packed appearance represents 590 the dense structure of these communities as they exchange frequent citations with other members 591 of the same community. The names of the influential authors in each cluster are displayed to 592 make it easy to identify the principal communities the network can be subdivided into. This was 593 achieved by applying the PageRank algorithm to the entire network and ranking the importance 594 of each node based on its PageRank value.

Daniel Gile had the highest PRA scores in the green cluster, the largest community in the dataset with 2,366 scholars. Authors such as Franz Pöchhacker, Jean Herbert, Roderick Jones, Robin Setton, Yang Chengshu, David Carroll, George Miller, John Andreson also belonged to this cluster. Most of these top influencers' works are concerned with cognitive psychology or psycholinguistics, which would appear to indicate that those disciplines have a dominant influence on CIS research. Most notably, George Miller's The Magic Number Seven, Plus or Minus Two, and John Anderson's Cognitive Psychology and Its Implications, received 4 and 8 incoming citations respectively, suggesting that the interdisciplinary approach to interpreting research is one favored by many a scholar. It should be noted here that this community features outliers, but further research would be required to ascertain why, alongside scholars whose particular focus is cognition, CIS authors cite writers such as Herbert and Jones, neither of whom specializes in cognitive science, though both have produced popular textbooks on interpreting techniques.

609

610 Only one of the top ten scholars in this cluster was Chinese - this was Yang Chengshu, who has 611 served as director of the Graduate School of Translation and Interpretation at Fu Jen Catholic 612 University in Taiwan since 2006. She received a total of 195 incoming citations from CIS 613 scholars, her two most often-cited works being Research into Interpreter Training (In614 Degree=240) and Types of and Rules for Syntactic Linearity in SI (In-Degree=2). Her high615 ranking in this cluster - 6th out of 2,366 scholars - is worthy of remark: given that Taiwan has a 616 somewhat different socio-cultural background from that of mainland China, and that her 
617 specialty is Japanese-Chinese interpreting, her great popularity among CIS scholars was 618 unexpected. Several factors may explain this: though Yang has worked exclusively at academic 619 institutions in Taiwan, she received her PhD in Interpreting Studies from BFSU under the 620 supervision of Wang Kefei in 2006. She also has close ties with scholars from the PRC, 621 evidenced by the fact that the second of her most frequently cited works mentioned above was 622 co-authored with Wan Hongyu, a scholar from Shanghai. In addition, the popularity of her other 623 most-cited work indicates that the general principles of interpreter training know no linguistic 624 boundaries.

625

\subsection{Most influential scholars}

627

The following 30 people were identified as the most influential Western scholars in CIS based 630

Table 2: The top 30 Western scholars in CIS by PRA measures

632

633 Gile, Lederer, and Seleskovitch head the table, indicating that their research is highly influential 634 among their Chinese colleagues. When it comes to degree centrality (DC) measures the ranking 635 has shifted slightly: Gile, cited by 1,077 Chinese scholars, continues on top, while Seleskovitch, 636 cited by 756 , now ranks second. She is trailed by Lederer, who was cited by 674 researchers. 637 Their ranking by weighted degree centrality (WDC) remained consistent with their DC ranking: 638 Gile scored 2,116, Seleskovitch 1,207 and Lederer 1,020. The first had a total of 75 papers and 639 books receive incoming citations. The most widely cited of his works was Basic Concepts and 640 Models for Interpreter and Translator Training, whose main contribution to the field is 641 theoretical, though it does have a number of empirical components (See Table 3). Seleskovitch 642 and Lederer formulated the Interpretive Theory of Translation, which stresses the importance of 643 disregarding the linguistic structure of the original when interpreting into a foreign language. 644 Despite both having published empirical research, almost all Chinese scholars exclusively cite 645 the translations of their non-empirical works such as Interpreting for International Conferences: 646 Problems of Language and Communication, Interpréter Pour Traduire, and Pédagogie 647 Raisonnée de l'Interprétation - these were among the earliest Western interpreting theories 
648 introduced into China. Their influence on CIS research is perhaps attributable to the wide 649 availability of the translated versions of their works. Upon further analysis, of the top 30 Western 650 researchers, their works alone were cited in translation; all the others were cited in the original 651 language - for example, though two of Gile's French papers received incoming citations, they 652 were both cited in French. The citation data revealed that 27 of Seleskovitch's works and 23 of 653 Lederer's received citations. Tables 3 and 4 list the top five most cited works of these two 654 authors:

Table 3: The most influential works of Daniel Gile

657

658

Table 4: The most influential works of Danica Seleskovitch

659

660

Table 5: The most influential works of Marianne Lederer

661

662 Being cited by a large number of scholars does not necessarily translate into a particular 663 author's having a high PageRank score or being perceived as highly influential by his confreres. 664 For instance, David Gerver was cited by 160 Chinese scholars and received 257 citations, 665 ranking 20th in terms of DC and 16th in WDC, but his ranking dropped to 40th when calculated 666 by the PRA. Gile's paper (2000) indicates that Gerver's work is also reasonably popular in the 667 West. There is no doubt that Gerver was an influential pioneer in introducing methodologies 668 from experimental psychology into interpreting research in the 1970s, but his work was heavily 669 criticized by working interpreters for a lack of ecological validity, his manner of selecting 670 research participants, and the methods he employed for evaluating their performances (Gile, 671 1994). This may explain his low PRA score, which indicates that the most influential Chinese 672 scholars seldom cited his work - most citations of him were made by less influential researchers 673 such as graduate students. The difficulty of accessing his publications in China may be another 674 factor that contributes to his low score. A similar situation was observed for the rankings of 675 Setton and Moser-Mercer: The former received the seventh most citations, as shown by his 676 WDC score, but ranked only 13th in terms of influence. Having received his $\mathrm{PhD}$ from the 677 Chinese University of Hong Kong in 1997 and subsequently taught in Taiwan and Shanghai, he 678 remained active in CIS up to the time of his departure from the Shanghai International Studies 679 University in 2011. While he published a number of texts on interpreting over the years, only his 
$680 \mathrm{PhD}$ dissertation, which was later published as a monograph, received much attention from the 681 CIS community; the other papers he wrote received fewer than 15 incoming citations each (See 682 table 6). Moser-Mercer was cited by 221 Chinese colleagues, receiving a total of 381 citations, 683 giving her a ranking of 12th in DC and 6th in WDC, but her position fell to 21st in the PRA. 684 Examination of her works revealed that 39 of them received citations. The most cited was 685 Bridging the Gap: Empirical Research in Simultaneous Interpretation-the first collected 686 volume on empirical research into interpreting, on which she worked as a co-editor (see Table 7). 687

Table 6: The most influential works of Robin Setton

689

690

Table 7: The most influential works of Barbara Moser-Mercer

691

692 Working interpreters not engaged in research are still highly influential among Chinese 693 academics. Roderick Jones is a case in point: only his monograph Conference Interpreting 694 Explained, a manual that provides tips and techniques for honing interpreting skills, received 695 citations. However, his PRA ranking was 6th, immediately after Franz Pöchhacker. The 696 professional's intuitive understanding of working interpreters is sometimes deeper and more 697 comprehensive than that gained by collecting and analyzing empirical data, which might explain 698 why he is highly cited by influential Chinese scholars. In addition, many may find his writing easy to relate to and his suggestions simple to follow in interpreting practice, contributing to his

701 wide popularity in the CIS community.

A few translation scholars, such as Eugene Nida, Peter Newmark and Mona Baker, were also reasonably influential among Chinese academics. The first is noted for his Dynamic Equivalence Theory in translation, and works of his such as Language, Culture, and Translating, Dynamic Equivalence in Translating, and Language and Culture: Context in Translating were widely cited by Chinese colleagues. This contributed to his PRA ranking of 4th among all Western scholars, behind Seleskovitch. He ranked 5th in DC, having been cited by 467 Chinese scholars, and received the same ranking for WDC with 730 citations. Though Pöchhacker outranked him in both DC and WDC, Nida's PRA score seems to suggest that he has a higher research impact than Pöchhacker in the CIS community. As mentioned earlier, the reason for the discrepancy between their DC/WDC and PRA scores is that Nida received more citations from influential 
712 CIS scholars than Pöchhacker, whereas the latter is more popular among low-ranking CIS 713 scholars.

715 In addition, Western scholars in linguistics, sociology, cognitive science and psychology played 716 an appreciable role in CIS research. For example, Lyle Bachman's research on language testing 717 was often cited in work on the assessment of interpreting competence. Dan Sperber, a sociologist 718 and cognitive scientist, developed the Relevance Theory in collaboration with Deidre Wilson, a 719 psychologist by training. This theory has been used by numerous Chinese scholars to shed light 720 on the processes of listening comprehension, note-taking and language production in 721 interpreting.

The data reveal the following to be the top 30 most influential Chinese scholars:

Table 8: Top 30 Chinese scholars in CIS by PRA

In comparison with their Western colleagues, the composition of the top-ranking Chinese scholars in PRA was quite homogenous. Of the top 30, no fewer than 25 (all but Mei Deming, Liu Miqing, Wu Bing, Hu Gengshen and Zhuang Enping) are interpreting scholars.

730

Cai Xiaohong ranked 5th in DC and 6th in WDC, but had the second highest PRA score of all, indicating that she had a very large research impact among her fellows. In 2000 she defended her doctoral dissertation, in which she studied the development of competence in consecutive interpreting by conducting an experiment with 12 participants of different skill levels. Since then she has published a number of monographs and papers, of which the top five most frequently cited are shown in Table 9. The present research revealed that as was the case for Moser-Mercer, Cai's most cited work was a collective volume which she co-edited. All the other top five works were research papers she has published over the years.

Table 9: The most influential works of Cai Xiaohong

742 Bao Gang ranked 1st in DC, having been cited by 543 people and receiving 592 citations, the 743 majority of them on his monograph An Overview of Interpreting Theories, but his ranking 
744 dropped to 4th in PRA. A possible explanation for this is that sadly he passed away in 1999 at 745 the age of 42: had he lived, he would surely have contributed a great deal more to CIS. In Table 746 10, which lists his top five most cited works, it is interesting to observe that the citation

747 distribution for his works is lopsided: the overwhelming majority of scholars cite his 748 aforementioned work, an introduction to the leading Western theories prevalent in the 1990s. A 749 possible explanation for this highly skewed distribution is, once again, his untimely death.

750

751

Table 10: The most influential works of Bao Gang

752

753

754

Liu Heping ranked 2nd in both DC and WDC, receiving a total of 843 citations from 513

755 scholars, but her ranking dropped to 8th in PRA. Her most cited works are shown in Table 11. Liu's incoming citations seem much more balanced than those of the previously mentioned CIS scholars. Though her monograph Interpreting Techniques: Scientific Thinking and Reasoning 757 received the majority of incoming citations, her papers, which occupied the rest of the top five spots, were also cited by numerous CIS scholars. Liu obtained her PhD in Translation Studies from the University of Paris III, and is an active member of the CIS community, regularly appearing as a keynote speaker at various conferences. A former student of Seleskovitch's, she continues to advocate the Interpretive Theory of Translation in her research.

762

763

Table 11: The most influential works of Liu Heping

764

765

As is the case for their Western colleagues, the opinions of Chinese practicing interpreters are 766 also highly valued by CIS scholars. Zhang Weiwei, who ranked 9th in PRA, once served as a staff interpreter at the United Nations' duty station in Geneva: he was cited almost exclusively for his handbook English-Chinese Simultaneous Interpreting, which highlights the use of segmentation and syntactic linearity with numerous practical examples. In addition, his high rankings in DC (9th) and WDC (12th) suggest that he was equally popular with scholars both influential and less so.

773 Zhang Wei's rankings in PRA, DC and WDC merited some investigation. He topped the list in 774 WDC, his ranking having been significantly boosted by his weighted out-degree centrality 775 (WODC) - he made a total of 854 citations of other scholar's works in all his papers and in his 
776 doctoral dissertation, far exceeding the second highest in WODC, Ren Wen, whose total was 777 557. However, in DC Zhang slipped to 6th position, and in PRA to 44th. It should also be noted 778 that he was the most productive CIS author, with 28 papers recorded in the data-set. The 779 discrepancy between his influence score and research productivity might be explained by his 780 being a relative newcomer to CIS, having received his $\mathrm{PhD}$ in 2007-it is common that the older 781 a work is, the larger its readership and the more it will have been cited. Another plausible reason 782 for the discrepancy is that his doctoral dissertation, in which he investigated the relationship 783 between simultaneous interpreting and working memory, was highly technical and used a 784 combination of experimental, observational and questionnaire-based research methods; the 785 unfamiliar techniques he used may have deterred other CIS authors from adopting his work. 786 Furthermore, the research carried out for Question 3 of the present study revealed that authors 787 writing about cognitive issues were slightly more likely to end up in the bottom PageRank 788 influence group than those writing on other topics.

789

790 There are also a number of scholars who, though trained and specializing in other disciplines, 791 occasionally venture into the field of interpreting research, namely Mei Deming, Hu Gengshen, 792 Liu Miqing, Wu Bing and Zhuang Enping. Mei topped the Chinese scholars' PRA rankings. He 793 is among the most prolific MA thesis advisors in China, and was involved in launching the 794 Shanghai Interpreting Exams. A PhD graduate in linguistics and rhetoric from the University of 795 Indiana, he has taught numerous classes on linguistics, public speaking and movie appreciation.

796 In the data-set there were no research papers on interpreting written by him alone, though he co797 authored a few with his doctoral students. In addition to all the above he was the editor-in-chief 798 for An Advanced Course in Interpreting, which was first published in 1996 (new editions in 799 2000, 2005 and 2011), and for An Intermediate Course in Interpreting, first published in 1998 800 (new editions in 2003, 2008 and 2010). These are the mandatory test preparation books for the 801 Shanghai Interpreting Exams, and other Chinese authors' citing of them contributed to his 802 becoming one of the most influential CIS authors in the country. The case of Wu Bing follows 803 the same lines: she is mainly cited for her textbook on Chinese-to-English interpreting. Neither 804 author has published any empirical studies on the subject. Zhuang Enping specializes in cross805 cultural communication, but he has also written articles on the principles of interpreting and how 806 the differences between Eastern and Western styles of communication affect it. His high degree 
807 of influence among Chinese scholars (17th in PRA) indicates that a good number of researchers

808 draw inspiration from Communication Theory.

809

810 Unlike the situation with the top 30 most influential Western scholars, the top 30 Chinese list 811 had only one Chinese scholar whose research was completely unrelated to interpreting. Chen 812 Siping, who ranked 29th in PRA, focused on the application of the Relevance Theory to reading 813 comprehension; her high ranking would seem to indicate that influential Chinese scholars

814 frequently used her work as the theoretical underpinnings for their research.

815

$816 \quad 6.3$ Research topics and academic influence

817

$818 \quad$ 6.3.1. Summary of significant findings

819

820

As stated in the methodology section, inferences drawn from linear regressions were somewhat 821 unsatisfactory: F-tests proved only the linear model for Out-Degree to be statistically significant.

822

823 As discussed then, we consequently turned to multinomial regressions for three influence 824 groups - low, middle and high — for each of the network measures: PageRank, In-Degree, Out825 Degree, and EigenVector Centrality. We delimited the group divisions by determining the cutoff 826 points that led to the most statistically significant regression models.

827

828 For each measure and each influence group we report below the themes that had statistically 829 significant coefficients.

830

831 Table 12: Statistically significant themes in multinomial regression for PageRank

832

833 Table 13: Statistically significant themes in multinomial regression for In-Degree 834 
837 The change in likelihood column shows that authors who had $1 \%$ more publications in the 838 listed theme were $x \%$ more or less likely to be in that influence group for that influence measure.

839 Taking PageRank (see Table 12) as an example: when authors wrote $1 \%$ more publications that

840 fell into the Cognitive theme category, they were $0.4 \%$ less likely to be part of the Medium-

841 ranking influence group. In other words, an author with no Cognitive keywords in $100 \%$ of his

842 publications would be $40 \%$ more likely to have medium PageRank than an author all of whose

843 keywords were Cognitive.

844

845 Although the linear regressions had low statistical power, the trends they predicted coincide 846 with the findings of the multinomial regressions (see sections 6.3.2 and 6.3.4 below for 847 numerical results). In particular both Cognitive and Language keywords increased an author's 848 Out-Degree measure. The multinomial regression analysis clarified how these keywords 849 increased an author's Out-Degree measure. In particular, Cognitive keywords increased an 850 author's likelihood of having high Out-Degree, while Language keywords decreased the 851 likelihood of his having low Out-Degree.

852

853 The Lasso multinomial regressions for keywords (see section 6.3.5) also supported the earlier 854 multinomial regression results for theme category analysis. The consistency of these analyses is 855 supported by the fact that the majority of the keywords, and the theme categories that these 856 keywords belong to, share the same correlation sign (either positive or negative) as the influence 857 measure group of the authors who wrote them. To illustrate with an example, the keyword 858 "Theory" was positively correlated with an author's placement in the High-ranking group of 859 PageRank, and the theme category of this keyword (Miscellaneous) was also found to be 860 positively correlated with an author's likelihood of belonging to that same High-ranking group. It 861 should also be acknowledged that the Lasso regression analysis failed to detect a couple of 862 findings from the theme category analysis. For example, the positive relationship between 863 Social-cultural keywords and the High In-Degree influence group, and the negative relationship 864 between Language keywords and the Low Out-Degree group, were not picked up by the Lasso 865 model. It is likely that those keywords were not significant on their own, but collectively they 866 contributed to boosting an author into a certain influence group. 
868 In sum, from the findings above it was found that the most influential authors are those who 869 write about Social-cultural and non-mainstream topics. In particular, authors whose papers cover 870 Theory are more likely to be placed in the High influence group than those who do not. In 871 addition, the analysis revealed that those writing about Cognitive issues, especially Sensory 872 Memory, are more likely to include a significant number of bibliographic references.

873

\subsubsection{Linear regression: A first approach to modeling the relationship between network} influence and memes

876

877 For the preliminary analysis four standard linear regressions of each of the metrics were

878 performed using the theme profiles as predictors. The fact that each theme profile was 879 normalized might result in prolific and non-prolific authors having very similar profiles.

880 Therefore number of papers published was added as an extra predictor. This was done to separate 881 the effects of an author's theme profile from his or her overall frequency of publishing. Table 15 882 shows the F-test $p$-values for each model.

883

884 Table 15: F-test for different network influence measures

885

886 The only regression model which turned out to be significant was Out-Degree. In general terms 887 this means that the topics an author writes about are a good predictor of the number of outgoing 888 citations in his papers. For example, when people write about cognitive issues, the coefficient of 889 this theme (see Table 16 below) suggests that they tend to have higher Out-Degree scores, 890 meaning that they cite more papers. In sum, the coefficients in Table 16 essentially indicate that 891 the technical nature of an author's papers can be a reliable predictor of his ranking in Out-Degree 892 measure. A possible explanation for this is that more technical papers require the researcher to 893 conduct extensive literature review in order to ease the reader into his own research. This often 894 translates into a large number of citations in his or her papers. 
895

896

Table 16: Linear regression output of themes on out-degree centrality measure

897

898 The coefficients of themes in Table 16 represent the changes that would occur in Out-Degree if 899 that particular theme proportion were increased by $1 \%$. For example, a 1\% shift in an author's 900 theme profile to Cognitive would result in a 0.08381 increase in Out-Degree. A similar $1 \%$ shift 901 to Language would result in a 0.06429 increase. It should be noted, however, that the coefficient

902 for the number of papers represents the amount of Out-Degree shifted as a result of increasing 903 the number of papers by 1 . More specifically, the coefficient for the number of papers indicates 904 that having one more paper published might corresponds to a 0.59 increase in predicted Out905 Degree for that particular author.

906

907 It should be noted that a coefficient for Training does not appear in the regression summary.

908 This is due to the profile normalization discussed earlier. If an author's theme profile is known

909 for any five terms, their score for the sixth theme can be precisely inferred by 1 - sum (scores for

9105 themes). Hence when an author's theme profile is parameterized to five dimensions, as is

911 necessary for computational reasons, it contains the same information as a full 6-theme profile.

912 Each theme profile group was treated as a numeric variable so that the effect of changing the

913 relative proportion of that theme would be clearly visible. Any positive coefficient in the

914 regression means that trading off Training keywords for keywords in that particular theme

915 profile results in an increased Out-Degree.

916

917 Relative effects can be revealed by using algebraic manipulations of the coefficient estimates

918 and adjusting for Training. For example, consider the impact of trading a percentage of the

919 Cognitive theme for the Language theme. The model loses 0.08 Out-Degree (that is, 0.08

920 outgoing citations, or $8 \%$ of one outgoing citation) by trading $1 \%$ of the Cognitive theme for $1 \%$ 
921 of the Training theme; it gains 0.06 Out-Degree by trading Training for Language. The following

922 formula shows how these values are computed:

923

924 Effect $(-1$ cognitive, +1 language $)=$ Effect $(-1$ cognitive,+1 training $)+$ Effect $(-1$ training,+1

925 language $)=-0.08+0.06=-0.02$.

926

927 Trading off $1 \%$ of Cognitive theme for $1 \%$ of Language theme lowers Out-Degree by 0.02 . If 928 this shift in themes were to be repeated 50 times, the model predicts that the paper would have its 929 Out-Degree score reduced by 1 (that is, one fewer outgoing citation).

930

931 The analysis thus reveals that using more Cognitive or Language themes in papers is a predictor 932 of having higher Out-Degree counts, i.e. authors writing on those subjects generate more 933 references. The data also suggest that having larger numbers of papers published is correlated, 934 although only marginally, with higher Out-Degree, an interesting finding in that one would 935 expect writing more papers to lead to an inevitable increase in the total number of outgoing 936 citations. There are two possible explanations for this marginal effect. Firstly, the fact that $30 \%$ 937 of the CIS papers in the data-set do not have any references suggests that a large proportion of 938 authors do not necessarily have a high number of outgoing citations, even if they have written 939 multiple publications. Secondly, it is possible that a few highly prolific authors generate only 940 small numbers of outgoing citations, which may have an effect on the correlation between the 941 number of publications and the Out-Degree measure.

942

943 It should be noted that the multiple and adjusted R-squared values in Table 16 are rather low-

944 less than $1 \%$ of the variance in the data is explained by the model, even though the $p$-values for 945 several predictors are very small. This means that the results of standard linear regression are not 946 very promising: while the trend detected is significant, the model still cannot explain the data 
947 very well. Huge disparities between authors' network influence measures were a likely cause for

948 this inadequacy. A large discrepancy or inequality in a response variable makes a linear

949 regression inadequate because estimated effects are highly influenced by a few extreme values,

950 and hence effects which pertain to the rest of the population are subsumed. In the following

951 section we examine this inadequacy further, and describe mathematically the amount of network

952 measure inequality through an analysis of Gini coefficients.

953

954 6.3.3 Gini Coefficient: Measuring disparities in each network measure across authors

955

956 The Gini Coefficient has typically been used to calculate income inequality in populations, by

957 converting the cumulative distribution of wages into a single number. In such cases a Gini value

958 of 0 corresponds to complete income equality, i.e. every individual is earning exactly the same

959 wage, whereas a value of 1 corresponds to complete inequality, i.e. one individual is in receipt of

960 all the wealth. For the present study the procedure was applied to evaluate the distribution of our

961 four network influence measures; the results are summarized in Table 17. Once the procedure

962 was completed, we matched these Gini coefficients to publicly reported wage distributions of

963 countries, allowing us to form analogies which demonstrate the amount of inequality in network

964 measures and hence the inadequacy of linear regression.

965

966 Table 17: Gini Coefficients for four network influence measures

967

968 The analysis revealed that PRA has the most equitable distribution of ranks, roughly equal to

969 the amount of wage inequality in Belgium ${ }^{10}$. On the other hand, the other ranks are much less

970 balanced, with larger inequality than the wage inequality in any country measured by the World

971 Bank - the one with the largest recorded Gini Coefficient is the Seychelles, with 0.658 in 2007.

10 All references to world income inequality calculations are taken from the World Bank's Gini index: http://data.worldbank.org/indicator/SI.POV.GINI/ 
972 Despite PRA's having a relatively equitable distribution of ranks, the PRA scores of CIS authors

973 were still not good enough for linear regression analysis: 200 authors (about 10\% of the CIS

974 total) control $30 \%$ of the scores, though the remaining $70 \%$ are almost perfectly equally

975 distributed among the remaining 1,950. This means that we are missing inference on the

976 remaining 1,950 authors because linear regression of the PageRank score focuses on the largest

977 difference, the one between the 1,950 authors and the top 200. This calls for a better approach to

978 evaluating the impact of an author's theme profile on his or her rankings.

979

980 6.3.4 Multinomial logistic regression: Procedure for stratifying authors into high-, middle-

981 and low-ranking groups

982

983 Though linear regression was the first choice - and most straightforward-method of

984 explaining authors' levels of influence based on their theme profiles and numbers of

985 publications, as we have seen it turned out to be lacking when it came to explaining their

986 influence within networks. An alternative approach was to classify the authors into three

987 influence groups—-low, middle and high —using multinomial regression.

988

989 The three groups can be defined in different ways depending on which cutoffs are used to

990 separate them. Only two parameters are needed to define the three groups: one cutoff value to

991 separate the low and middle groups and another to separate the middle and the high. N.B.: By

992 knowing these two values we can know both the length and the midpoint value of the middle

993 influence group.

994

995 Figure 6: $P$-value of the deviance test for a significance multinomial regression over all 209 possible PageRank

996 group divisions. The divisions are parameterized by the midpoint of the Middle Ranking group on the $\mathrm{x}$ axis and by

997 its width on the y axis. Darker squares denote more significant regressions and should be regarded as indicating that

998 these divisions are significantly influenced by theme profile.

999 
1000 The multinomial models represent the probability of an author's being in each of the three

1001 groups, given an author's theme profile and number of papers written. The coefficients of each

1002 of the multinomial models were determined by fitting each model to the data, where the response

1003 variable is now an indicator of each author's allocation to the three groups corresponding to each

1004 model. The coefficients depended on how the three groups were divided, and each pair of cutoffs

1005 was associated with a different multinomial model. In Figures 6 and 7, each square represents a

1006 model and is thus associated with a certain definition of the three groups of authors, or

1007 equivalent to certain values of the two cutoffs.

1008

1009 Figure 6 shows the results of fitting each of the 209 group divisions for PageRank, and

1010 calculating a $p$-value for the null hypothesis of no significant relation between theme profile and

1011 group membership. Since we expect about 21 groups to have p-values less than 0.1 completely

1012 by chance, it is not instructive to report all groups with low $p$-values as indicative of significance.

1013 Many of these low p-values will not relate to significant effects and would confuse results.

1014

1015 Instead, the quality of a group of models was evaluated by a so-called False Discovery Rate

1016 (FDR) analysis. In the present study the FDR of a group of models is the expected proportion of

1017 models that are not good, i.e. are not statistically significant ones, therefore the lower the FDR

1018 the better the group of models. The quality of each model was measured by a quantity called $q$ -

1019 value. Simply put, to build a group of models with an FDR lower than a certain threshold value,

1020 only models with a $q$-value lower than that value can be included. The results of this FDR

1021 analysis for PageRank are summarized in Figure 7. $p$-value. It is the minimal FDR at which the test result is considered significant. The best pair of cutoffs for PageRank are the 20th percentile for low-ranking authors and the 85th for high-ranking ones. 
1028 Figure 7 shows that the FDR-based approach makes it easy to identify groups of good models:

1029 there are three stripes of squares associated with a small FDR. The furthest (red) stripe to the

1030 right corresponds to the models whose group divisions yield the most statistically significant

1031 results, i.e. which best explain the connection between an author's theme profile and his

1032 influence.

1033

1034 The red squares correspond to $q$-values lower than 0.05 , so the group of models corresponding

1035 to the red squares has an FDR below 5\%. In other words, no more than 5\% of these models are

1036 expected to be non-significant. Similarly, since the orange squares correspond to $q$-values above

10370.05 and below 0.10 , all groups of models, whether they correspond only to orange squares or to

1038 orange and red ones, have an FDR below 10\%. The same reasoning can be repeated for each

1039 color of square and its associated $q$-values. In Figure 5 the three diagonal stripes of red and

1040 orange squares correspond to the best and relatively good models described in this paragraph.

1041

1042 Once groups of relatively good models (each defined by a pair of cutoffs) had been determined,

1043 the selection of a 'stand-out' model, i.e. one with an exceptionally good pair of cutoffs, was still

1044 required. A clustering procedure known as k-medoids, by which similar pairs of cutoffs are

1045 divided into different groups, was employed at this point (Kaufman \& Rousseeuw, 1987). The

1046 chosen divisions corresponded to the most central point in the cluster with the smallest FDR (i.e.

1047 the cluster with the best statistical significance). The best pair of cutoffs for PageRank are the

1048 20th percentile for low-ranking authors and the 85th for high-ranking ones (see Figure 7).

1050 Similar analysis revealed the optimal cutoffs for In-Degree to be $[0.6,0.95]$ and for Out-Degree $1051[0.6,0.8]$. Theme profiles best explain the differences between the bottom $60 \%$ of Out-Degrees 1052 (less than two incoming citations), the top 5\% (more than 33 incoming citations), and the middle 1053 group (those between 60 and 95\%). Similarly they best explain the differences between the 
1054 bottom $60 \%$ of In-Degrees ( 0 outgoing citations), the top 20\% of Out-Degrees (more than 24

1055 outgoing citations), and the middle group. Note that for Out-Degree we can choose any cutoff

1056 below $64 \%$ as that percentage of authors had 0 Out-Degree citations. Hence any such quantile

1057 choice would result in absolute cutoffs of 0 and 24 outgoing citations. For EigenVector no

1058 cutoffs were found that had a chance of less than $20 \%$ of being non-null hypotheses - in fact no

1059 significant $p$-values at all came to light. As a result the predictors worked better with PageRank

1060 than EigenVector for measuring author influences in the CIS network; for this reason the latter

1061 measurement was not adopted for the following analysis. Table 18 shows optimal cutoff

1062 quantiles and their corresponding measure values:

1063

1064 Table 18: Optimal cutoff quantiles and their measure values

1065 The output shown in Tables 19,20 and 21 below summarizes the results of multinomial 1066 regression of theme profiles on the three network measures. The coefficients are symmetric 1067 relative odds on the log scale.

1068

1069 We present all of our results on the relative odds scale, which can be found from the 1070 transformation $\exp ($ coef $)$. This makes it easier to compare the relative effect of different theme 1071 profile variables in different subgroups. For example, a coefficient of -0.004 for cognitive in the 1072 middle group for PageRank means that authors who have 1\% less cognitive articles have on 1073 average $1-\exp (-0.004)=0.006$ percent smaller odds. It is straightforward to transform odds

1074 into raw percentages by taking into account the intercept coefficient and an author's theme 1075 profile. Consider an author who has 100\% cognitive articles. They would have exp (0.988 - 100 $* .004)=1.8$ to 1 odds, or about a $1.8 /(1+1.8)=0.62$ chance of being in the middle group.

1077

1078 For PageRank it was found that trading off $1 \%$ of Cognitive for $1 \%$ of any other paper gave us $1079 \exp (-0.004)=0.996$ lower odds, or roughly a $0.4 \%$ lower likelihood of being in the middle 
1080 group of PageRanks. The same trade-off for Socio-cultural gave us exp $(-0.006)=0.994$ lower

1081 odds, or roughly a $0.6 \%$ lower likelihood. The only positive significant relationship between

1082 theme profile and placement in the high PageRank group came from trading for Miscellaneous

1083 papers. Every percentage point increase in Miscellaneous papers gave roughly 1.007 greater odds

1084 of being in the top $15 \%$ of PageRanks. This is attributable to the fact that some highly influential

1085 papers were classified as Miscellaneous. Earlier research (Xu, 2014 \& 2015) indicated that the

1086 Miscellaneous theme accounts for only $9 \%$ of the total themes mentioned in MA theses, $0 \%$ in

1087 doctoral dissertations and $5.1 \%$ in research papers, meaning that non-mainstream topics have

1088 been explored by few CIS researchers, whereas numerous papers on Cognitive, Language,

1089 Professional and Socio-cultural issues have competed for attention. It appears to be much easier

1090 for those authors with papers on Miscellaneous subjects to get noticed and be cited by other

1091 researchers.

1092

1093 Table 19: PageRank Multinomial Regression

1095 Another way of describing a 0 coefficient for a theme profile variable for a certain group is as 1096 follows: when one percentage point of this variable is exchanged for one of the Training theme, 1097 there is no change in the probability of an author's belonging to the group under consideration.

1098 For example, when an author trades $1 \%$ of Cognitive or Language for $1 \%$ of Training, the 1099 probability of his belonging to the high-ranking PageRank group is not affected at all, but that 1100 probability is reduced by 0.007 when he trades $1 \%$ of Miscellaneous for $1 \%$ Training theme.

1102 The next item to be scrutinized was the regression on In-Degree. The In-Degree multinomial 1103 results showed that trading off any theme profile for Language papers gave 1.006 higher odds of 1104 being in the middle In-Degree group (greater than 2 but fewer than 33 incoming citations). It was 1105 also observed that if an author traded off any theme profile for the Socio-cultural themes, they 1106 would have 1.011 higher odds to be in the the high In-Degree group (having more than 33 1107 incoming citations). This was the most significant effect of any theme on any group, suggesting 
1108 that authors on Socio-cultural issues are easily identified and cited by their confreres. As was the

1109 case for the Miscellaneous theme, Socio-cultural issues received little - though slightly greater-

1110 attention from CIS researchers and authors: $10.7 \%$ for research papers, $11.1 \%$ for MA theses and

$111112.4 \%$ for doctoral dissertations. Socio-cultural issues play an important part in interpreting,

1112 which can all too easily be affected by factors such as contexts and hidden power relations

1113 between various actors in the dialogue (Pym, Shlesinger \& Jettmarová, 2006).

1114

1115 Table 20: In-Degree Multinomial Regression

1116

1117 Finally, the same procedure was applied for Out-Degree measure. Here it was observed that if 1118 authors wrote more Language papers, they were $\exp (-0.004)=0.996$, or had 0.004 lower odds to 1119 end up generating the least number of citations (low rank group). It should be noted, however, 1120 that since the $p$-value in this case was 0.09 , this finding is marginal at best. In addition, an author 1121 had a 1.008 higher probability of having between 1 and 24 outgoing citations (middle rank 1122 group) by writing more Professional papers, 1.009 higher odds of at least 24 citations (high rank 1123 group) by writing more Cognitive papers, and 1.077 higher odds of at least 24 outgoing citations 1124 by writing more papers in general. These results were found generally to tally with those of the 1125 linear regression described in Section 6.3.2.

1126

Table 21: Out-Degree Multinomial Regression

1128

1129

6.3.5 Regularized multinomial regression for predicting influence by keywords

1130 A regularization technique called Lasso was run for multinomial regression (Tibshirani, 1996)

1131 with 10-fold cross-validation to approximate the optimal set of keywords which were truly

1132 significant. The Lasso works by applying a penalty to the absolute value of coefficients,

1133 providing a principled way to set the coefficients of non-significant keywords to 0. Any

1134 remaining keywords were considered to be significantly related to the network measure. For the 
1135 dependent variables the same optimal cutoffs were used as those found by the FDR analysis

1136 examined in Table 18. By this technique, keywords that were not relevant enough for the present

1137 analysis could be discarded.

1139 Only the positive or negative character of each significant keyword's association with influence

1140 is given in Table 22. It was decided to show only the signs of the coefficients rather than their

1141 values, because it is difficult to interpret coefficient values modified by applying the Lasso

1142 method. However, the method retains the signs of the coefficients, which makes it relevant to

1143 show their signs.

1144

1145 Table 22: PageRank Keyword Profile Regression

1146

1147 The PageRank model (see Table 22) summarizes the most significant keywords and the role

1148 they play in deciding which theme group a particular author is most likely to belong to. Both

1149 Attention and Nominalization were correlated with authors belonging to the low influence group,

1150 suggesting that those who write about these two topics tend to end up with low influence as

1151 measured by PageRank. This finding coincides with the earlier theme profile analysis, where

1152 both Cognitive (the theme for Attention) and Language (the theme for Nominalization) issues

1153 were positively correlated with the low influence group. At the other end of the spectrum,

1154 Theory was positively correlated with authors in the high influence group, suggesting that

1155 scholars writing in that vein were very likely to receive high PageRank scores, a finding in line

1156 with the earlier one that Miscellaneous themes are positively correlated with the high influence

1157 group-Theory belongs to the Miscellaneous category.

1159 The second model indicates that 20 keywords were significantly associated with the In-Degree

1160 measure. The largest effect was that Language-related words were more likely to be found in the

1161 Middle group, which coincides with the theme profile analysis. However, the Lasso regression 
1162 was not able to detect that Socio-cultural themes predicted placement in the High In-Degree

1163 group. A possible explanation for this is that many Socio-cultural words were not significant on

1164 their own but collectively contributed to boosting an author into the high influence group.

1165

1166 Table 23: In-Degree Keyword Profile Regression

1167

1168 The third model indicates that three keywords were significantly associated with the Out-

1169 Degree measure. As found in both the theme profile cutoff and linear regressions, Cognitive

1170 words were positively correlated with being in the High Out-Degree group. Unlike the theme

1171 profile regressions, the model found that a Professional word correlated with the High group but

1172 it did not pick up on the impact of Language words.

1173

1174

Table 24: Out-Degree Keyword Profile Regression

1175

1176

1177

1178

1179

1180

1181

1182

1183

1184 'classic' textbooks that contain the established fundamentals of the subject. All of which

1185 indicates that the CIS community is a diverse one, with scholars forming into groups based on

1186 their shared characteristics. Despite this diversity, a small number of individuals stood out as the

1187 most influential. While the top 30 Western authors exerting the most influence in CIS had a wide 
1188 range of distinct areas of interest and expertise, all but one of the Chinese top 30 specialized in

1189 research into interpreting. It is also worth noting that a substantial proportion of the overall total

1190 of researchers had several of their works cited, while a minority was influential thanks to only

1191 one or two publications.

1192

1193 This paper also contributes to better understanding how research topics are associated with a

1194 CIS author's influence. It concluded that authors writing about non-mainstream topics (i.e.

1195 Miscellaneous themes) were more likely to be found in the high-influence PageRank group than

1196 those tackling 'bread-and-butter' subjects, and those writing on Socio-cultural themes were more

1197 likely to be placed in the high-influence In-Degree group. The study also identified several

1198 keywords significantly correlated with an author's network measures: Theory (high-influence

1199 PageRank group); Attention and Nominalization (low-influence PageRank); Language-related

1200 keywords (middle-ranking In-Degree); and Sensory Memory and Trade Association (high-

1201 ranking Out-Degree). The findings for keywords were broadly in line with those for themes,

1202 suggesting that authors who wish to make their mark in the academic community would do well

1203 to embrace certain topics while avoiding others.

1205 When Interpreting Studies was in its infancy in the 1960s only a handful of isolated authors, 1206 scattered throughout the world, were conducting research (Gile, 2013a); today, despite its still 1207 relative youth in comparison with 'old timers' such as linguistics, philology, etc., it is well on the 1208 way to becoming a mature discipline in its own right (Moser-Mercer, 2011), and China's 1209 contribution to its rapid development has undoubtedly been considerable. The aim of this 1210 scientometric survey has been to provide a panorama of the evolution of Chinese Interpreting

1211 Studies while demonstrating the merits of blending traditional citation analysis with Social

1212 Network Analysis to produce such a survey. It is hoped that its findings might help authors better

1213 appreciate the trade-offs they need to make when choosing research topics and the potential 
1214 academic influence that may result from their choices, as well as, more importantly, offering

1215 policy-makers new insights and food for thought as they chart the future course of CIS research.

1216

1217

1218

1219

1220

1221

1222

1223

1224

1225

1226

1227

1228

1229

1230

1231

1232

1233 


\section{References}

1235 Anderson, J. R. (1980). Cognitive psychology and its implications. San Francisco: W.H.

1236 Freeman.

1237 Bao, G. (1992). Preparatory training for undergraduate interpreting students (In Chinese). Chinese Translators Journal, 6, 23-25.

Bao, G. (1996). Methods for memorizing terms before an assignment (In Chinese). Journal of Beijing International Studies University, 3(71), 42-45.

Bao, G. (1998). An overview of interpreting theories (In Chinese). Beijing: Travel Education Press.

Bao, G. (1999). Reasoning and comprehension in the interpreting process (In Chinese). Journal of Beijing International Studies University, 1(87), 1-12.

Bao, G. (1998). Semantic issues in the interpreting process (In Chinese). Journal of Beijing International Studies University, 4(84), 103-110.

Baumgartner, H., \& Pieters, R. (2003). The structural influence of marketing journals: A citation analysis of the discipline and its subareas over time. Journal of Marketing, 67(2), 123-139.

Buela-Casal, G., Zych, I., Medina, A., Jesus, M. I., Lozano, S., \& Torres, G. (2009). Analysis of the influence of the two types of the journal articles; theoretical and empirical on the impact factor of a journal. Scientometrics, 80(1), 265-282.

Cai, X. (2001). Interpretation study with an interdisciplinary perspective (In Chinese). Chinese Translators Journal, 22(2), 26-29.

Cai, X. (2001). The process of consecutive interpreting and skills development (In Chinese). Contemporary Foreign Languages, 24(3), 277-284.

Cai, X. (2002). An exploration of interpreting research (In Chinese). Hong Kong: Maison d'Edition Quaille.

Cai, X. (2003). Assessing interpreting quality: An approach based on units of meaning (In Chinese). Journal of Foreign Languages, 5(147), 75-80.

Cai, X. (2007). Interpreting assessment (In Chinese). Beijing: China Translation \& Publishing Corporation.

Chen, P., \& Redner, S. (2010). Community structure of the physical review citation network. Journal of Informetrics, 4(3), 278-290. 
1265 Delisle, J., \& Woodsworth, J. (Eds.). (1995). Translators through history. Amsterdam: John 1266 Benjamins.

1267 Fortunato, S. (2010). Community detection in graphs. Physics Reports, 486(3), 75-174.

1268 Gambier, Y., Gile, D., \& Taylor, C. (Eds.). (1997). Conference interpreting: Current trends in research. Amsterdam: J. Benjamins.

Gao, B. (2008). Conjectures and refutations (In Chinese) (Unpublished doctoral dissertation). Shanghai International Studies University.

Gile, D. (1994). Opening up in interpretation studies. In M. Snell-Hornby, F. Pöchhacker, \& K. Benjamins.

Gile, D. (1995). Basic concepts and models for interpreter and translator training. Amsterdam:

$$
\text { J. Benjamins. }
$$

Gile, D. (1997). Conference interpreting as a cognitive management problem. In J. Danks, G. Publications.

Gile, D. (1999). Use and misuse of the literature in interpreting research. The Interpreters' Newsletter, 9, 29-43.

Gile, D. (2000). The history of research into conference interpreting: A scientometric approach. Target, 12(2), 297-321.

Gile, D. (2013a). Institutional, social and policy aspects of research into conference interpreting. Comares.

Gile, D. (2013b). Scientificity and theory in translation studies. In Y. Gambier \& L. V. Doorslaer (Eds.), Handbook of Translation Studies (Vol. 4, pp. 148-155). John Benjamins Publishing Company. doi:10.1075/hts.4 
1295 Gile, D., Dam, H. V., Dubslaff, F., Martinsen, B., \& Scholdager, A. (Eds.). (2001). Getting started in interpreting research: Methodological reflections, personal accounts and advice for beginners. Amsterdam: John Benjamins.

Hachul, S., \& Jünger, M. (2005). Drawing large graphs with a potential-field-based multilevel algorithm. Lecture Notes in Computer Science, 3383, 285-295.

Haslam, N., Ban, L., Kaufmann, L., Loughnan, S., Peters, K., Whelan, J., \& Wilson, S. (2008). What makes an article influential? Predicting impact in social and personality psychology. Scientometrics, 76(1), 169-185.

Hsieh, F. Y., Bloch, D. A., \& Larsen, M. D. (1998). A simple method of sample size calculation for linear and logistic regression. Statistics in Medicine, 17(14), 1623-1634.

Hu, G., \& Sheng, Q. (2000). Another decade of CIS research (In Chinese). Chinese Science \& Technology Translators Journal, 13(2), 39-44.

Hurley, N., \& Rickard, S. (2009). Comparing Measures of Sparsity. IEEE Transactions on Information Theory, 55(10), 4723-4741.

Jiang, G. (2008). From Price to Rousseau: International figures that influence the scientometric development in China (In Chinese). Evaluation and Management, 6(3), 1-5.

1312 Kaufman, L., \& Rousseeuw, P. J. (1987). Clustering by means of Medoids. In Y. Dodge (Ed.), Statistical data analysis based on the L1-norm and related methods (pp. 405-416). North-Holland.

Kuhn, T. S. (1970). The structure of scientific revolutions, ( $2^{\text {nd }}$ ed.). Chicago: University of Chicago Press.

Lambert, S., \& Moser-Mercer, B. (Eds.). (1994). Bridging the gap: Empirical research in simultaneous interpretation. Amsterdam: John Benjamins.

Lederer, M. (1994). La traduction aujourd'hui: Le modèle interprétative. Paris: Hachette F.L.E. 
1325 Liu, H. (2001). Interpreting techniques: Scientific thinking and reasoning (In Chinese). Beijing: China Translation \& Publishing Corporation.

1327 Liu, H. (2001). Interpreting theories and teaching of today and tomorrow (In Chinese). Chinese Translators Journal, 22(2), 17-18.

Liu, H. (2002). A few thoughts on standardized interpreter teaching plans (In Chinese). Chinese Translators Journal, 23(3), 56-58.

Liu, H. (2005). Trends in interpreting research (In Chinese). Chinese Translators Journal, 26(4), 71-74.

1333 Liu, S., \& Wang, L. (2007). A survey of interpreting research in China over the past ten years (In Chinese). Journal of Guangdong University of Foreign Studies, 18(1), 37-40.

Liu, Z. (1999). Hongzhou Zhao and Scientometrics in China (In Chinese). Studies in Science of Science, 17(4), 104-109.

Lowry, P. B., Karuga, G. G., \& Richardson, V. J. (2007). Assessing leading institutions, faculty, and articles in premier information systems research journals. Communications of the Association for Information Systems, 20, 142-203.

Mei, D. (1996). An advanced course in interpreting (In Chinese) (1st ed.). Shanghai: Shanghai Foreign Education Press.

Mei, D. (1998). An intermediate course in interpreting (In Chinese) (1st ed.). Shanghai: Shanghai Foreign Language Education Press.

Moser-Mercer, B. (1996). Quality in interpreting: Some methodological issues. The Interpreters' Newsletter, 7, 43-55.

Moser-Mercer, B. (1997). Beyond curiosity: Can interpreting research meet the challenge? In J. Danks, G. Shreve, G. M. Fountain, \& M. McBeath (Eds.), Cognitive processes in translation and interpretation (pp. 176-195). London: Sage Publications. 
1355 Moser-Mercer, B. (1997). Process models in simultaneous interpretation. In C. Hauenschild \& S.

1356

1357

1358

1359

1360

1361

1362

1363

1364

1365

1366

1367

1368

1369

1370

1371

1372

1373

1374

1375

1376

1377

1378

1379

1380

1381

1382

1383

1384 Heizmann (Eds.), Maschine Translation and Translation Theory (pp. 3-18). Hague: Mouton de Gruyter.

Moser-Mercer, B. (2011). Identifying and interpreting scientific phenomena: Simultaneous challenges to interpreting research. In B. Nicodemus \& L. Swabey (Eds.), Advances in Interpreting Research: Inquiry in action Vol. 11, (pp. 47-58). John Benjamins Publishing Company.

Mu, L., \& Wang, B. (2009). Interpreting studies in China: A journal articles-based analytical survey (In Chinese). Chinese Translators Journal, 4, 19-25.

Nederhof, A. J. (2006). Bibliometric monitoring of research performance in the Social Sciences and the Humanities: A Review. Scientometrics, 66(1), 81-100.

Newman, M., \& Girvan, M. (2004). Finding and evaluating community structure in networks. Physical Review E, 69(2), 1-16.

Newman, M. (2001). Scientific collaboration networks. I. Network construction and fundamental results. Physical Review E, 64(1), 016131-1-016131-8.

Nida, E. A. (1993). Language, culture, and translating. Shanghai: Shanghai Foreign Language Education Press.

Nida, E. A. (1995). Dynamic equivalence in translating. In An Encyclopedia of translation: Chinese-English English-Chinese [C/Z] (pp. 223-230). Hong Kong: The Chinese University Press.

Nida, E. A. (2001). Language and culture: Contexts in translating. Shanghai: Shanghai Foreign Language Education Press.

Otte, E., \& Rousseau, R. (2002). Social network analysis: A powerful strategy, also for the information sciences. Journal of Information Science, 28(6), 441-453.

Pym, A., Shlesinger, M., \& Jettmarová, Z. (2006). Sociocultural aspects of translating and interpreting. Amsterdam: John Benjamins.

Ravallion, M., \& Wagstaff, A. (2011). On measuring scholarly influence by citations. Scientometrics, 88(1), 321-337.

Reichardt, J., \& Bornholdt, S. (2004). Detecting fuzzy community structures in complex networks with a Potts model. Physical Review Letters, 93(21), 1-4. 
1385 Rényi, A., \& Erdős, P. (1959). On random graphs. Publicationes Mathematicae, 6(290-297),

1386

1387

1388

1389

1390

1391

1392

1393

1394

1395

1396

1397

1398

1399

1400

1401

1402

1403

1404

1405

1406

1407

1408

1409

1410

1411

1412

1413

1414

5.

Ruan, P. (2012). Assessment of research programs in Chinese hospitals (In Chinese). Chinese Hospitals, 16(3), 46-49.

Schaeffer, S. E. (2007). Graph clustering. Computer Science Review, 1(1), 27-64.

Schneider, A. M. (2009). Four stages of a scientific discipline; four types of scientist. Trends in Biochemical Sciences, 34(5), 217-223.

Seleskovitch, D., \& Lederer, M. (1984). Interpréter pour traduire (In French). Paris: Didier érudition.

Seleskovitch, D., \& Lederer, M. (1989). Pédagogie raisonnée de l'interprétation (In French). Paris: Didier erudition.

Seleskovitch, D., \& Lederer, M. (1995). A systematic approach to teaching interpretation. The Registry of Interpreters for the Deaf.

Seleskovitch, D. (1978). Interpreting for international conferences. Washington: Pen and Booth.

Seleskovitch, D. (1978). Language and cognition. In NATO conference series, 6, (pp. 333-341). Springer US.

Setton, R., \& Moser-Mercer, B. (2000). The Geneva (ETI) perspective on interpretation research. Conference Interpretation and Translation, 2.

Setton, R. (1994). Experiments in the application of discourse studies to interpreter training. In C. Dollerup \& A. Lindegaard (Eds.), Teaching Translation and Interpreting 2.

Setton, R. (1998). Meaning assembly in simultaneous interpretation. Interpreting, 3(2), 163-199.

Setton, R. (1999). Simultaneous interpretation: A cognitive-pragmatic analysis. Amsterdam: Benjamins.

Setton, R. (2006). New demands on interpreting and the learning curve in interpreter training. In Proceedings of the 5th National Conference and International Forum on Interpreting (pp. 36-71). Shanghai: Shanghai Foreign Language Education Press.

Takeda, Y., \& Kajikawa, Y. (2010). Tracking modularity in citation networks. Scientometrics, 83(3), 783-792.

Tang, F. (2010). Empirical research in Chinese Interpreting Studies (In Chinese). Foreign Language World, 137(2), 39-46. 
1415 Tang, S., \& Zhou, Y. (1958). The nature of interpreting (In Chinese). Western Languages, 2(3), 321-327.

1417 Tibshirani, R. (1996). Regression shrinkage and selection via the lasso. Journal of the Royal Statistical Society: Series B (Statistical Methodology), 58(1), 267-288.

1419 Tsien, H. (1977). Modern science and technology. People's Daily.

1420 Van Dalen, H. P., \& Henkens, K. (2001). What makes a scientific article influential? The case of demographers. Scientometrics, 50(3), 455-482.

1422 Wan, H., \& Yang, C. (2005). Types of and rules for syntactic linearity in SI. Chinese Translators Journal, 26(3)

Wang, R. (2006). Simultaneous interpretation and ITS professionalization in China (In Chinese). In Proceedings of the 5th National Conference and International Forum on Interpreting (pp. 143-154). Shanghai: Shanghai Foreign Language Education Press.

Wang, Y., Wu, Y., Pan, Y., Ma, Z., \& Rousseau, R. (2005). Scientific collaboration in China as reflected in co-authorship. Scientometrics, 62(2), 183-198.

$\mathrm{Xu}, \mathrm{Z}$. (2014). Chinese Interpreting Studies: Genesis of a discipline. Forum: International Journal of Interpretation and Translation, 12(2), 159-190.

Xu, Z. (2015). The past, present and future of Chinese MA theses in Interpreting Studies: A scientometric survey. Perspectives. doi:10.1080/0907676X.2015.1011175

Yang, C. (2005). Research into Interpreter Training (In Chinese). Beijing: China Translation \& Publishing Corporation.

Zhang, H., \& Zhang, Y. (1997). Scientometric study on research performance in China (In Chinese). Information Processing \& Management, 33(1), 81-89.

Zhang, W. (1999). English-Chinese simultaneous interpreting (In Chinese). Beijing: China Translation \& Publishing Corporation.

Zhang, W. (2008). A study of the effect of simultaneous interpreting on working memory's growth potential (In Chinese). Modern Foreign Languages, 31(4), 423-430.

Zhang, W. (2011). A contrastive analysis of Chinese and western interpreting research (In Chinese). Foreign Languages in China, 5, 94-106.

1443 Zhao, H., \& Jiang, G. (1985). Shifting of world's scientific center and scientists' social ages. 
Data organization framework using the idea behind SQL relational data management system.

Arrows between tables show examples of unique pieces of information which are indexed in multiple tables, as a way of linking data points between tables.

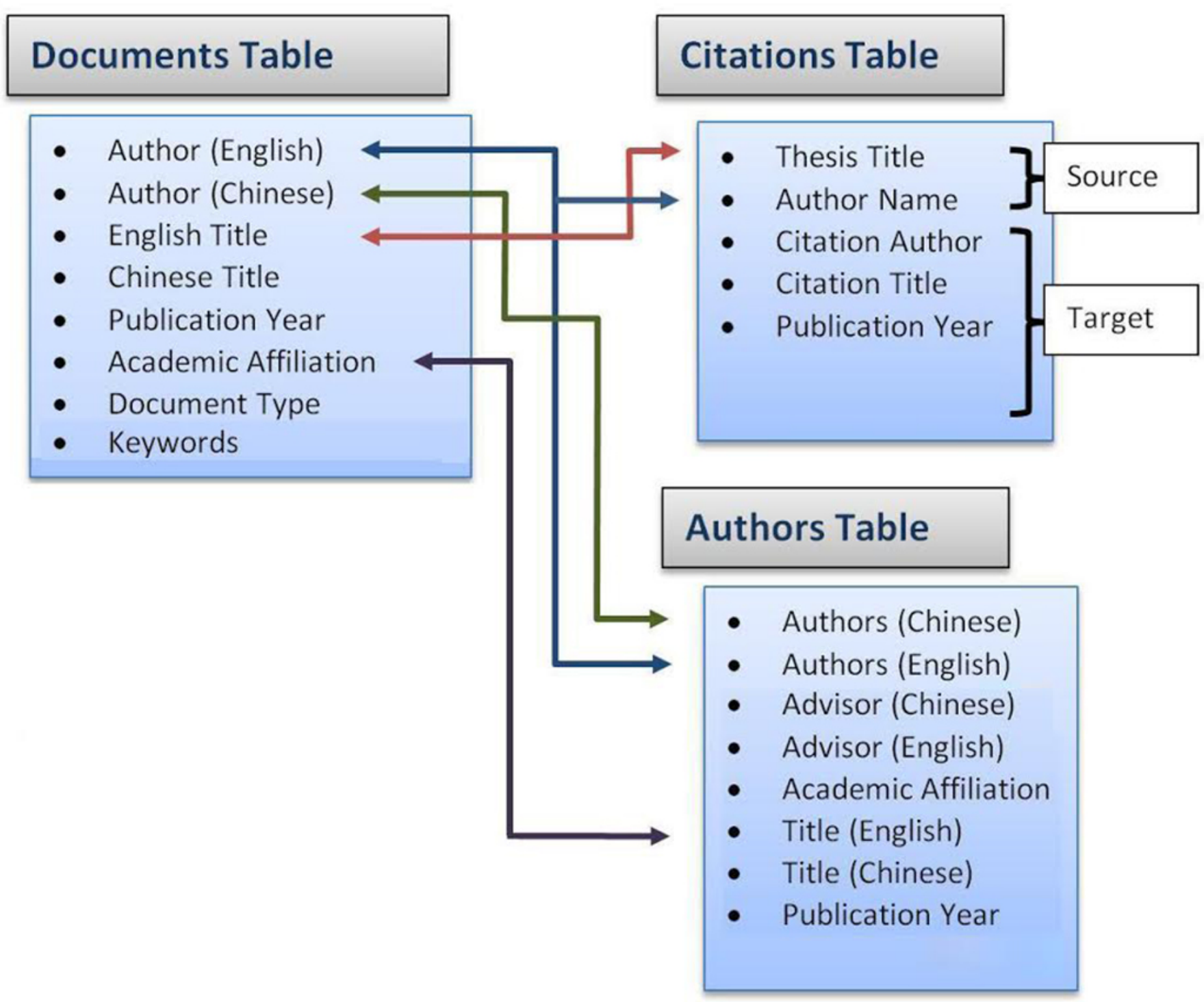


2

Ranking group cutoffs

$5 \%$ of all authors are in the low-ranking group, $15 \%$ in the high-ranking, and the remaining $80 \%$ in the middle-ranking.

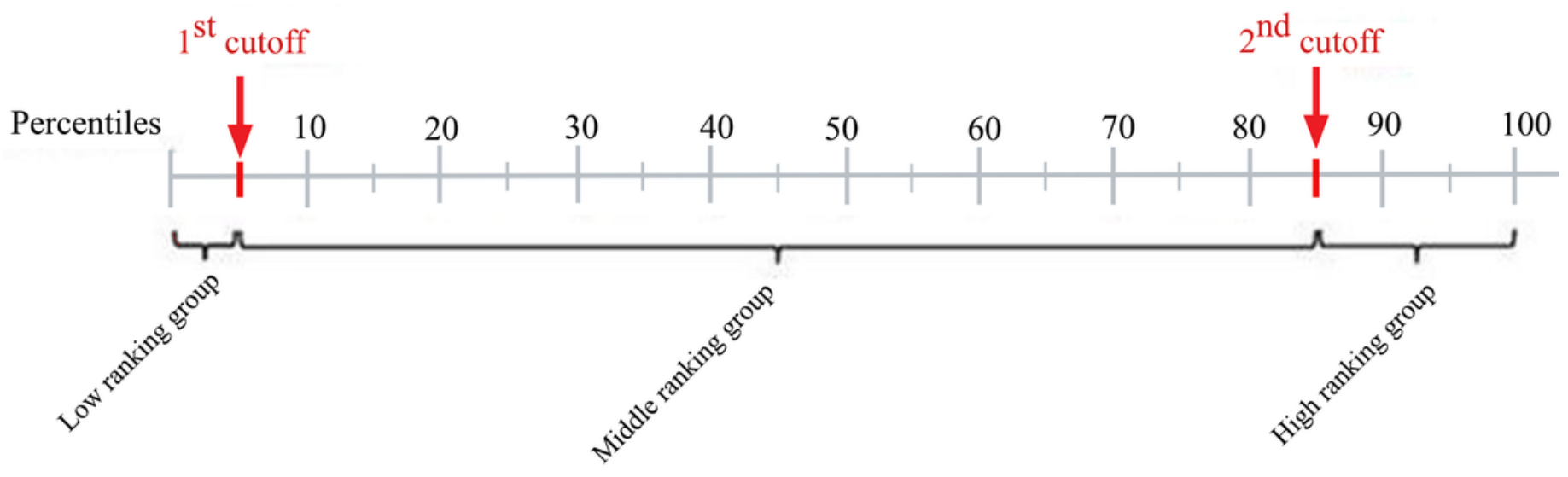




\section{3}

CIS citation network visualization

Entire CIS network laid out using a force-directed algorithm

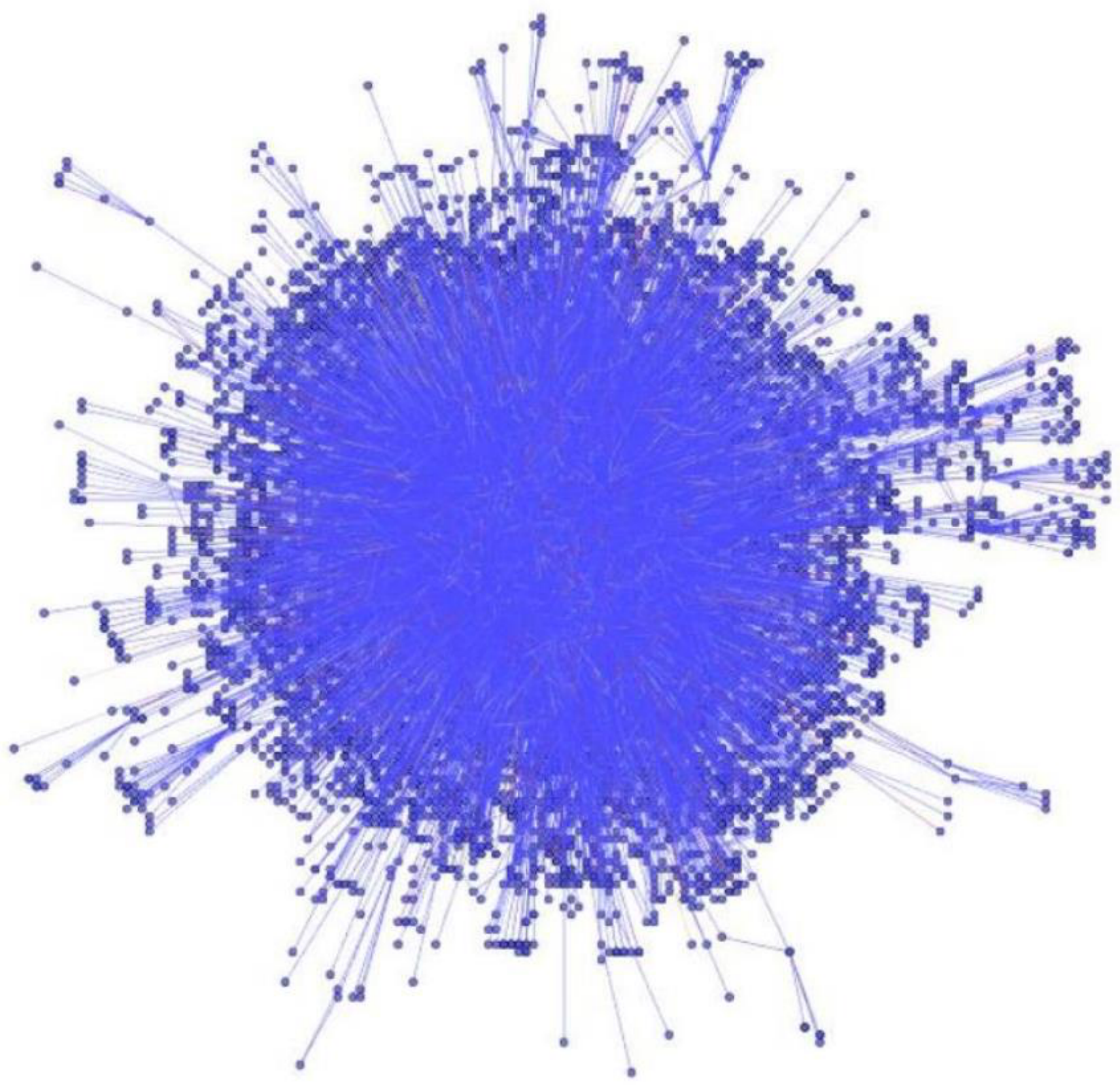


4

Top $150 \mathrm{CIS}$ authors

Studying the behavior of interconnections of the top 150 authors. Nodes are placed using a circular algorithm where the most important nodes are displayed in the center to highlight top ranked authors.

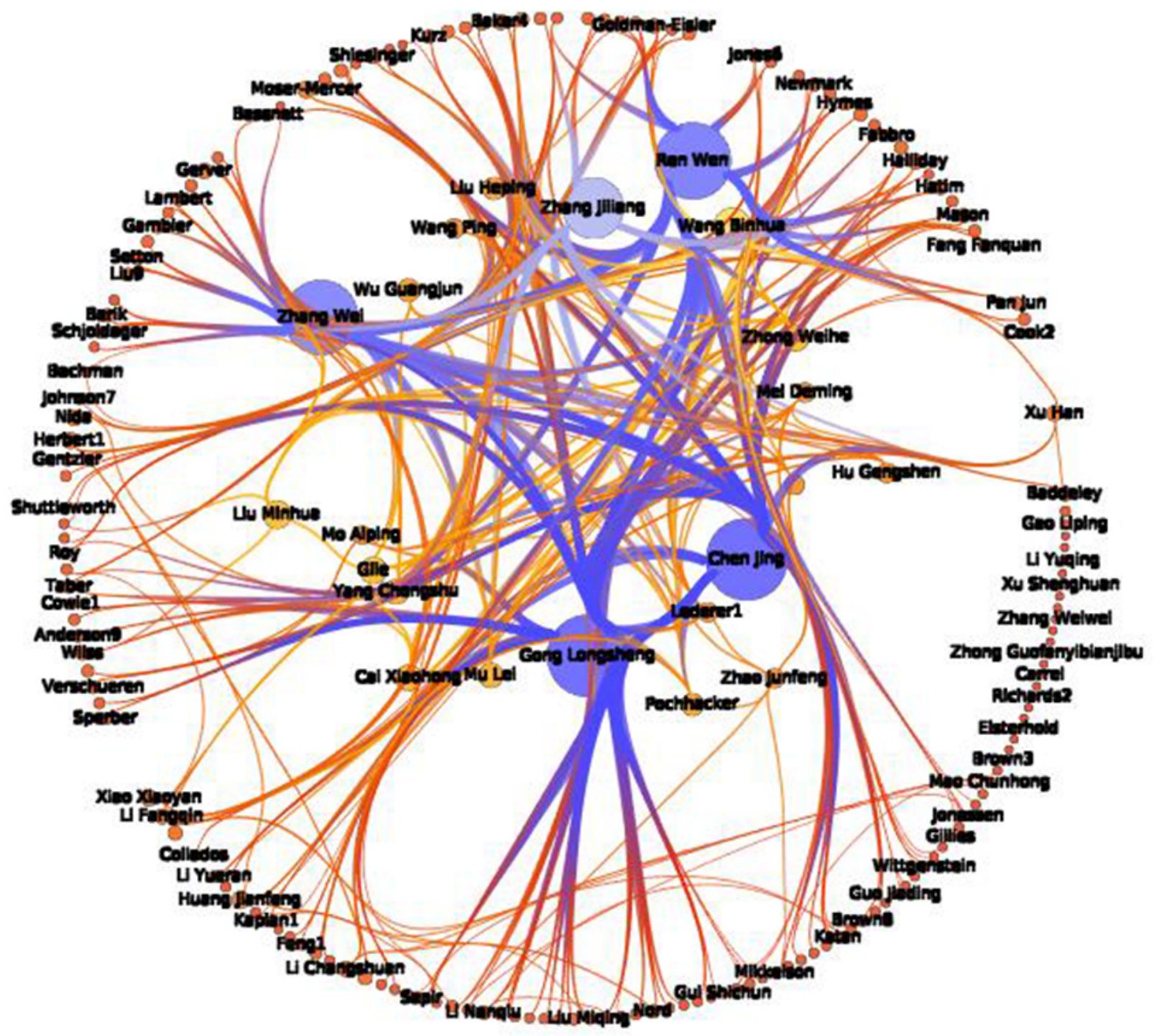


5

\section{CIS communities}

Seven communities in CIS obtained by the Spin-glass algorithm
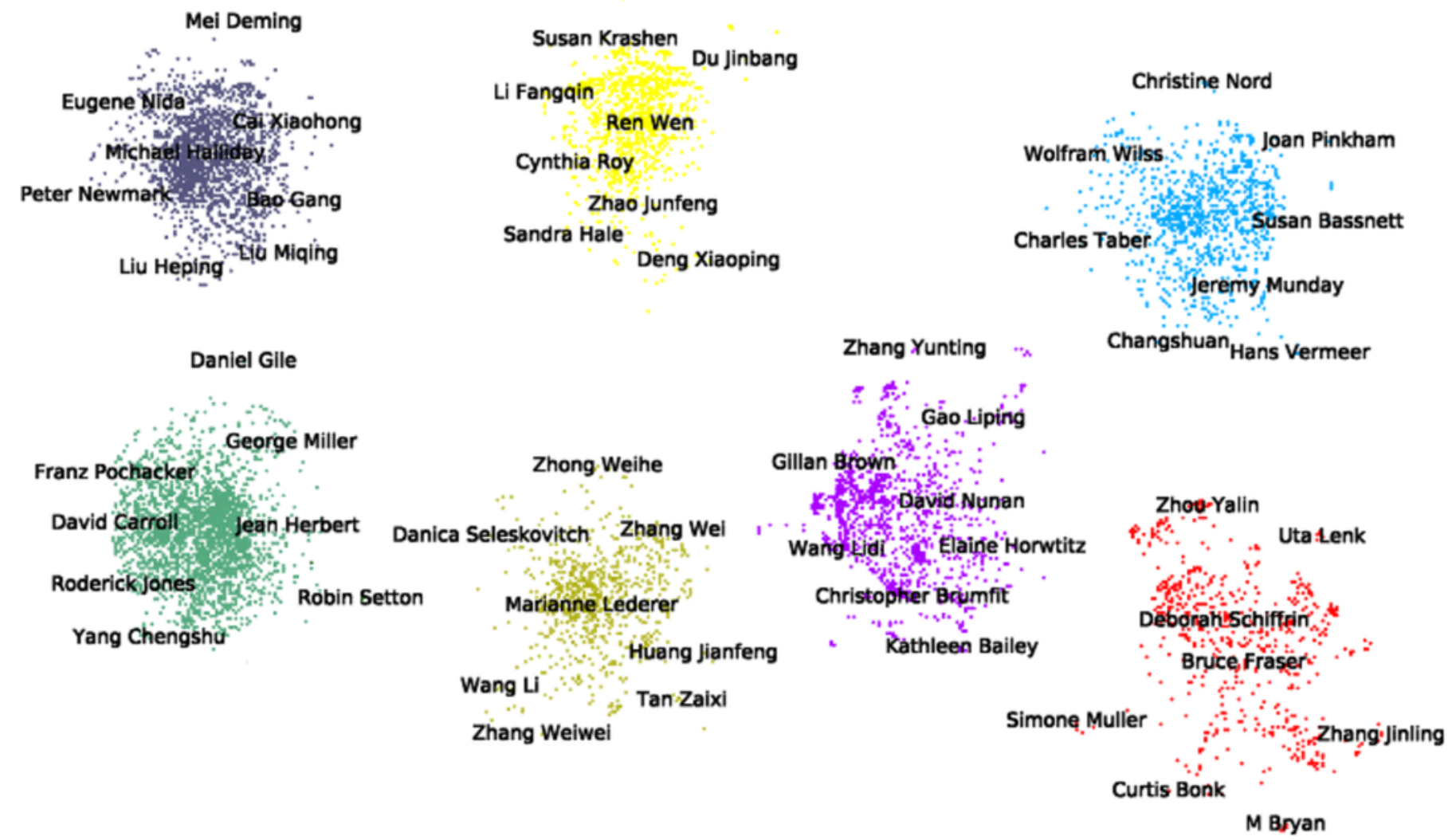


\section{6}

$P$-value of the deviance test for a significance multinomial regression over all 209 possible PageRank group divisions

The divisions are parameterized by the midpoint of the Middle Ranking group on the $\mathrm{x}$ axis and by its width on the y axis. Darker squares denote more significant regressions and should be regarded as indicating that these divisions are significantly influenced by theme profile.

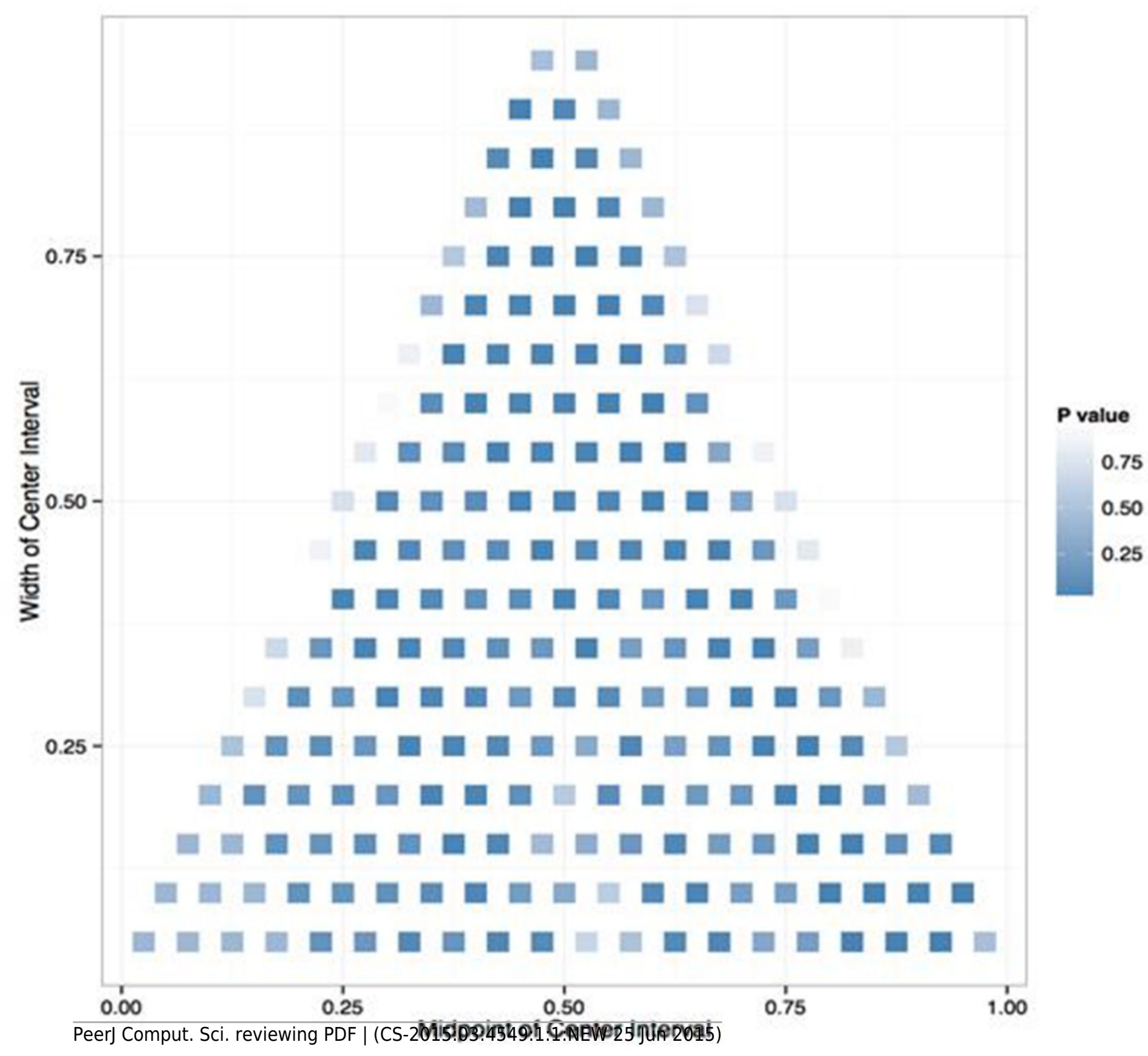


7

$q$-values for PageRank influences

The $q$-value is a False Discovery Rate (FDR) analysis analogue of the $p$-value. It is the minimal FDR at which the test result is considered significant. The best pair of cutoffs for PageRank are the 20th percentile for low-ranking authors and the 85th for high-ranking ones.

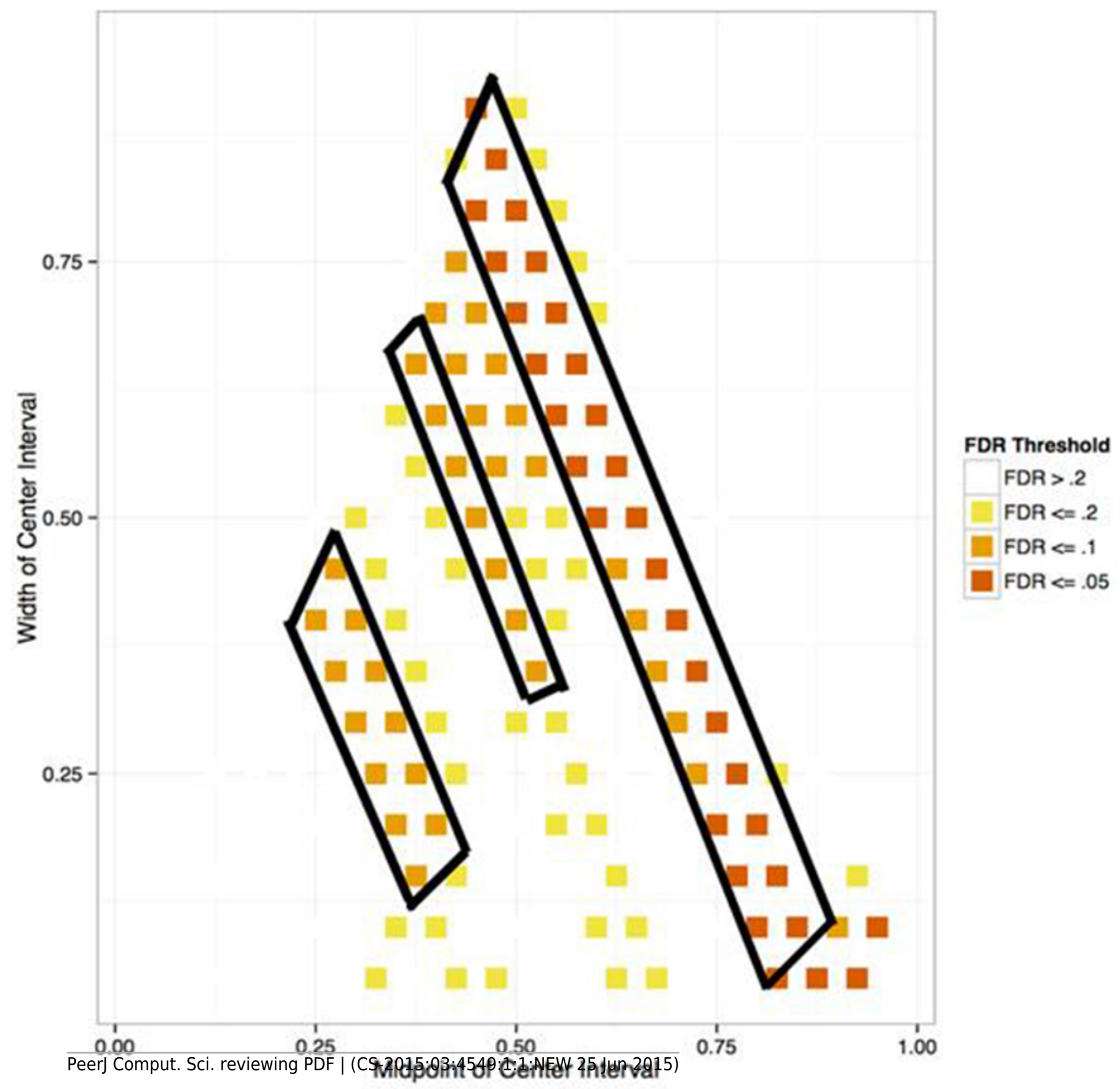




\section{Table $\mathbf{1}$ (on next page)}

Comparison of different clustering algorithms using CIS data 
1

\begin{tabular}{|l|l|l|}
\hline Clustering Algorithms & $\begin{array}{l}\text { Number of Clusters } \\
\text { Generated }\end{array}$ & Modularity \\
\hline Louvain & 20 & 0.416 \\
\hline FastGreedy & 87 & 0.387 \\
\hline Walktrap & 693 & 0.266 \\
\hline Leading Eigen Vector & 2 & 0.241 \\
\hline Infomap & 643 & 0.339 \\
\hline Label Prop & 76 & 0.062 \\
\hline Spin-glass & 7 & 0.434 \\
\hline
\end{tabular}

2 Table 1: Comparison of different clustering algorithms using CIS data 


\section{Table 2 (on next page)}

The top 30 Western scholars in CIS by PRA measures 


\begin{tabular}{|c|c|c|c|c|c|c|c|c|}
\hline No. & PageRank & $\begin{array}{l}\text { Author's } \\
\text { Name }\end{array}$ & $\begin{array}{l}\text { In- } \\
\text { Degree }\end{array}$ & $\begin{array}{l}\text { Out- } \\
\text { Degree }\end{array}$ & Degree & $\begin{array}{l}\text { Weighted } \\
\text { Degree }\end{array}$ & $\begin{array}{l}\text { Weighted } \\
\text { In-Degree }\end{array}$ & $\begin{array}{l}\text { Weighted } \\
\text { Out-Degree }\end{array}$ \\
\hline 1 & 0.00773432 & Daniel Gile & 1077 & 0 & 1077 & 2116 & 2116 & 0 \\
\hline 2 & 0.00496506 & $\begin{array}{l}\text { Marianne } \\
\text { Lederer }\end{array}$ & 674 & 0 & 674 & 1020 & 1020 & 0 \\
\hline 3 & 0.00487847 & $\begin{array}{l}\text { Danica } \\
\text { Seleskovitch }\end{array}$ & 756 & 1 & 757 & 1208 & 1207 & 1 \\
\hline 4 & 0.00251794 & Eugene Nida & 467 & 0 & 467 & 730 & 730 & 0 \\
\hline 5 & 0.00230936 & $\begin{array}{l}\text { Franz } \\
\text { Pochhacker }\end{array}$ & 538 & 0 & 538 & 903 & 903 & 0 \\
\hline 6 & 0.00169282 & $\begin{array}{l}\text { Roderick } \\
\text { Jones }\end{array}$ & 277 & 0 & 277 & 310 & 310 & 0 \\
\hline 7 & 0.00158975 & Jean Herbert & 225 & 0 & 225 & 262 & 262 & 0 \\
\hline 8 & 0.00114476 & $\begin{array}{l}\text { Peter } \\
\text { Newmark }\end{array}$ & 271 & 0 & 271 & 360 & 360 & 0 \\
\hline 9 & $9.87 \mathrm{E}-04$ & Christine Nord & 166 & 0 & 166 & 212 & 212 & 0 \\
\hline 10 & $9.34 \mathrm{E}-04$ & $\begin{array}{l}\text { Michael } \\
\text { Halliday }\end{array}$ & 204 & 0 & 204 & 323 & 323 & 0 \\
\hline 11 & 7.92E-04 & Mona Baker & 241 & 0 & 241 & 338 & 338 & 0 \\
\hline 12 & 7.22E-04 & Herbert Grice & 83 & 0 & 83 & 101 & 101 & 0 \\
\hline 13 & 7.11E-04 & Robin Setton & 271 & 0 & 271 & 376 & 376 & 0 \\
\hline 14 & $6.80 \mathrm{E}-04$ & $\begin{array}{l}\text { Deirdre } \\
\text { Wilson }\end{array}$ & 175 & 0 & 175 & 244 & 244 & 0 \\
\hline 15 & 6.77E-04 & Dan Sperber & 174 & 0 & 174 & 207 & 207 & 0 \\
\hline 16 & $6.71 \mathrm{E}-04$ & \begin{tabular}{|l} 
Jef \\
Verschueren
\end{tabular} & 58 & 0 & 58 & 79 & 79 & 0 \\
\hline 17 & $6.21 \mathrm{E}-04$ & David Carroll & 201 & 0 & 201 & 221 & 221 & 0 \\
\hline 18 & $6.16 \mathrm{E}-04$ & Gerald Miller & 103 & 0 & 103 & 114 & 114 & 0 \\
\hline 19 & $6.05 \mathrm{E}-04$ & John Anderson & 106 & 0 & 106 & 138 & 138 & 0 \\
\hline 20 & 5.99E-04 & $\begin{array}{l}\text { Miriam } \\
\text { Shlesinger }\end{array}$ & 236 & 0 & 236 & 356 & 356 & 0 \\
\hline 21 & $5.54 \mathrm{E}-04$ & $\begin{array}{l}\text { Barbara } \\
\text { Moser-Mercer }\end{array}$ & 221 & 0 & 221 & 381 & 381 & 0 \\
\hline
\end{tabular}




\begin{tabular}{|l|l|l|l|l|l|l|l|l|}
\hline 22 & $5.31 \mathrm{E}-04$ & Roger Bell & 130 & 0 & 130 & 137 & 137 & 0 \\
\hline 23 & $5.10 \mathrm{E}-04$ & $\begin{array}{l}\text { Wolfram } \\
\text { Wilss }\end{array}$ & 132 & 0 & 132 & 150 & 150 & 0 \\
\hline 24 & $4.97 \mathrm{E}-04$ & Basil Hatim & 204 & 0 & 204 & 272 & 272 & 0 \\
\hline 25 & $4.89 \mathrm{E}-04$ & $\begin{array}{l}\text { Ernst-August } \\
\text { Gutt }\end{array}$ & 132 & 0 & 132 & 162 & 162 & 0 \\
\hline 26 & $4.88 \mathrm{E}-04$ & Jenny Thomas & 53 & 0 & 53 & 62 & 62 & 0 \\
\hline 27 & $4.77 \mathrm{E}-04$ & $\begin{array}{l}\text { Christian } \\
\text { Matthiessen }\end{array}$ & 71 & 0 & 71 & 80 & 80 & 0 \\
\hline 28 & $4.75 \mathrm{E}-04$ & $\begin{array}{l}\text { Claire } \\
\text { Kramsch }\end{array}$ & 76 & 0 & 76 & 82 & 82 & 0 \\
\hline 30 & $4.49 \mathrm{E}-04$ & $\begin{array}{l}\text { Mark } \\
\text { Shuttleworth }\end{array}$ & 85 & 0 & 85 & 92 & 231 & 0 \\
\hline
\end{tabular}

2 Table 2: The top 30 Western scholars in CIS by PRA measures 


\section{Table 3 (on next page)}

The most influential works of Daniel Gile 
1

\begin{tabular}{|l|c|}
\hline \multicolumn{1}{|c|}{ Cited Works } & PageRank Scores \\
\hline Basic Concepts and Models for Interpreter and Translator Training & 0.004854 \\
\hline Conference Interpreting as a Cognitive Management Problem & $2.15 \mathrm{E}-04$ \\
\hline Conference Interpreting: Current Trends in Research & $1.27 \mathrm{E}-04$ \\
\hline Opening up in Interpretation Studies & $1.19 \mathrm{E}-04$ \\
\hline Getting Started in Interpreting Research & $6.62 \mathrm{E}-05$ \\
\hline
\end{tabular}

2 Table 3: The most influential works of Daniel Gile

3 


\section{Table 4(on next page)}

The most influential works of Danica Seleskovitch 
1

\begin{tabular}{|l|c|}
\hline \multicolumn{1}{|c|}{ Cited works } & PageRank Scores \\
\hline Pédagogie Raisonnée de l'Interprétation & $3.48 \mathrm{E}-04$ \\
\hline A Systematic Approach to Teaching Interpretation & $2.44 \mathrm{E}-04$ \\
\hline Language and Cognition & $1.06 \mathrm{E}-04$ \\
\hline Interpréter Pour Traduire & $4.27 \mathrm{E}-05$ \\
\hline Translation of L'Interprète dans les conférences internationales & $4.27 \mathrm{E}-05$ \\
\hline
\end{tabular}

2 Table 4: The most influential works of Danica Seleskovitch

3 


\section{Table 5(on next page)}

The most influential works of Marianne Lederer 
1

\begin{tabular}{|l|c|}
\hline \multicolumn{1}{|c|}{ Cited works } & PageRank Scores \\
\hline La traduction aujourd'hui: le modèle interprétatif & $6.18 \mathrm{E}-04$ \\
\hline Pédagogie Raisonnée de l'Interprétation & $3.48 \mathrm{E}-04$ \\
\hline A Systematic Approach to Teaching Interpretation & $2.44 \mathrm{E}-04$ \\
\hline Simultaneous Interpretation: Units of Meaning and Other Features & $1.31 \mathrm{E}-04$ \\
\hline Interpréter Pour Traduire & $4.27 \mathrm{E}-05$ \\
\hline
\end{tabular}

2 Table 5: The most influential works of Marianne Lederer

3 


\section{Table 6 (on next page)}

The most influential works of Robin Setton 
1

\begin{tabular}{|l|c|}
\hline \multicolumn{1}{|c|}{ Cited Work } & PageRank Scores \\
\hline Simultaneous Interpretation: A Cognitive-pragmatic Analysis & $4.99 \mathrm{E}-04$ \\
\hline $\begin{array}{l}\text { New Demands on Interpreting and the Learning Curve in } \\
\text { Interpreter Training }\end{array}$ & $5.67 \mathrm{E}-05$ \\
\hline Meaning Assembly in Simultaneous Interpreting & $5.45 \mathrm{E}-05$ \\
\hline $\begin{array}{l}\text { Experiments in the Application of Discourse Studies to } \\
\text { Interpreter Training }\end{array}$ & $5.45 \mathrm{E}-05$ \\
\hline The Geneva (ETI) Perceptive on Interpretation Research & $4.27 \mathrm{E}-05$ \\
\hline
\end{tabular}

2 Table 6: The most influential works of Robin Setton 


\section{Table 7 (on next page)}

The most influential works of Barbara Moser-Mercer 
1

\begin{tabular}{|l|c|}
\hline \multicolumn{1}{|c|}{ Cited Work } & PageRank Scores \\
\hline Quality in Interpreting: Some Methodological Issues & $1.23 \mathrm{E}-04$ \\
\hline $\begin{array}{l}\text { Bridging the Gap: Empirical Research in Simultaneous } \\
\text { Interpretation }\end{array}$ & $9.75 \mathrm{E}-05$ \\
\hline $\begin{array}{l}\text { Beyond Curiosity: Can Interpreting Research Meet the } \\
\text { Challenge }\end{array}$ & $8.98 \mathrm{E}-05$ \\
\hline $\begin{array}{l}\text { Simultaneous Interpretation: A Hypothetical Model and its } \\
\text { Practical Application }\end{array}$ & $8.83 \mathrm{E}-05$ \\
\hline Process Models in Simultaneous Interpretation & $7.55 \mathrm{E}-05$ \\
\hline
\end{tabular}

2 Table 7: The most influential works of Barbara Moser-Mercer

3 


\section{Table 8 (on next page)}

Top 30 Chinese scholars in CIS by PRA 


\begin{tabular}{|c|c|c|c|c|c|c|c|c|}
\hline No. & PageRank & $\begin{array}{l}\text { Author's } \\
\text { Name }\end{array}$ & $\begin{array}{c}\text { In- } \\
\text { Degree }\end{array}$ & $\begin{array}{l}\text { Out- } \\
\text { Degree }\end{array}$ & Degree & $\begin{array}{l}\text { Weighted } \\
\text { Degree }\end{array}$ & $\begin{array}{l}\text { Weighted } \\
\text { In-Degree }\end{array}$ & $\begin{array}{l}\text { Weighted } \\
\text { Out-Degree }\end{array}$ \\
\hline 1 & 0.00243402 & $\begin{array}{l}\text { Mei } \\
\text { Deming }\end{array}$ & 474 & 8 & 482 & 594 & 585 & 9 \\
\hline 2 & 0.00213418 & $\begin{array}{l}\text { Cai } \\
\text { Xiaohong }\end{array}$ & 407 & 8 & 415 & 582 & 573 & 9 \\
\hline 3 & 0.00137312 & Mu Lei & 125 & 9 & 134 & 160 & 148 & 12 \\
\hline 4 & 0.00119857 & Bao Gang & 543 & 0 & 543 & 592 & 592 & 0 \\
\hline 5 & 0.00119057 & Chen Jing & 110 & 183 & 293 & 388 & 147 & 241 \\
\hline 6 & 0.00114121 & Li Yuqing & 10 & 1 & 11 & 12 & 11 & 1 \\
\hline 7 & 0.0011069 & $\begin{array}{l}\text { Zhong } \\
\text { Weihe }\end{array}$ & 315 & 19 & 334 & 478 & 443 & 35 \\
\hline 8 & 0.00106898 & Liu Heping & 513 & 0 & 513 & 843 & 843 & 0 \\
\hline 9 & $9.33 \mathrm{E}-04$ & $\begin{array}{l}\text { Zhang } \\
\text { Weiwei }\end{array}$ & 326 & 0 & 326 & 328 & 328 & 0 \\
\hline 10 & 8.91E-04 & Liu Miqing & 415 & 0 & 415 & 498 & 498 & 0 \\
\hline 11 & $6.69 \mathrm{E}-04$ & Wu Bing & 102 & 0 & 102 & 107 & 107 & 0 \\
\hline 12 & $6.59 \mathrm{E}-04$ & $\begin{array}{l}\text { Zhong } \\
\text { Shukong }\end{array}$ & 305 & 0 & 305 & 311 & 311 & 0 \\
\hline 13 & $6.11 \mathrm{E}-04$ & $\begin{array}{l}\text { Yang } \\
\text { Chengshu }\end{array}$ & 195 & 30 & 225 & 283 & 247 & 36 \\
\hline 14 & $6.00 \mathrm{E}-04$ & $\begin{array}{l}\mathrm{Hu} \\
\text { Gengshen }\end{array}$ & 159 & 13 & 172 & 250 & 237 & 13 \\
\hline 15 & $5.96 \mathrm{E}-04$ & Ke Keer & 37 & 0 & 37 & 37 & 37 & 0 \\
\hline 16 & 4.91E-04 & $\begin{array}{l}\text { Zhang } \\
\text { Jiliang }\end{array}$ & 99 & 164 & 263 & 524 & 123 & 401 \\
\hline 17 & $4.26 \mathrm{E}-04$ & $\begin{array}{l}\text { Zhuang } \\
\text { Enping }\end{array}$ & 52 & 2 & 54 & 60 & 58 & 2 \\
\hline 18 & $4.26 \mathrm{E}-04$ & Sheng Qian & 93 & 6 & 99 & 107 & 101 & 6 \\
\hline 19 & $4.00 \mathrm{E}-04$ & Liu Minhua & 105 & 87 & 192 & 249 & 116 & 133 \\
\hline 20 & $3.93 \mathrm{E}-04$ & Li Yueran & 53 & 0 & 53 & 60 & 60 & 0 \\
\hline 21 & $3.61 \mathrm{E}-04$ & $\begin{array}{l}\text { Xiao } \\
\text { Xiaoyan }\end{array}$ & 178 & 14 & 192 & 219 & 199 & 20 \\
\hline
\end{tabular}




\begin{tabular}{|l|l|l|l|l|l|l|l|l|}
\hline 22 & $3.56 \mathrm{E}-04$ & $\begin{array}{l}\text { Hu } \\
\text { Qingping }\end{array}$ & 7 & 3 & 10 & 10 & 7 & 3 \\
\hline 23 & $3.51 \mathrm{E}-04$ & $\begin{array}{l}\text { Zhang } \\
\text { Junting }\end{array}$ & 10 & 0 & 10 & 11 & 11 & 0 \\
\hline 24 & $3.35 \mathrm{E}-04$ & $\begin{array}{l}\text { Li } \\
\text { Changshua } \\
\text { n }\end{array}$ & 85 & 12 & 97 & 118 & 105 & 13 \\
\hline 25 & $3.25 \mathrm{E}-04$ & $\begin{array}{l}\text { Yi } \\
\text { Honggen }\end{array}$ & 32 & 0 & 32 & 33 & 33 & 0 \\
\hline 26 & $3.19 \mathrm{E}-04$ & $\begin{array}{l}\text { Mu } \\
\text { Yuanyuan }\end{array}$ & 22 & 0 & 22 & 22 & 22 & 0 \\
\hline 27 & $3.19 \mathrm{E}-04$ & Pan Jun & 22 & 0 & 22 & 22 & 22 & 0 \\
\hline 28 & $3.17 \mathrm{E}-04$ & $\begin{array}{l}\text { Wang } \\
\text { Dawei }\end{array}$ & 109 & 3 & 112 & 128 & 125 & 3 \\
\hline 29 & $3.01 \mathrm{E}-04$ & $\begin{array}{l}\text { Chen } \\
\text { Siqing }\end{array}$ & 7 & 0 & 7 & 7 & 7 & 0 \\
\hline 30 & $2.88 \mathrm{E}-04$ & Li Nanqiu & 82 & 2 & 84 & 87 & 85 & 2 \\
\hline
\end{tabular}

2 Table 8: Top 30 Chinese scholars in CIS by PRA 


\section{Table 9 (on next page)}

The most influential works of Cai Xiaohong 


\begin{tabular}{|l|l|c|}
\hline \multicolumn{1}{|c|}{$\begin{array}{c}\text { Cited Work } \\
\text { (original Chinese title) }\end{array}$} & \multicolumn{1}{|c|}{ Englished Title } & PageRank Scores \\
\hline 以跨学科的视野拓展口译研究 & $\begin{array}{l}\text { Interpretation Study with an } \\
\text { Interdisciplinary Perspective }\end{array}$ & $4.32 \mathrm{E}-04$ \\
\hline 交替传译过程及能力发展 & $\begin{array}{l}\text { The Process of Consecutive } \\
\text { Interpreting and Skills Development }\end{array}$ & $1.77 \mathrm{E}-04$ \\
\hline 论口译质量评估的信息单位 & $\begin{array}{l}\text { Assessing Interpreting Quality: An } \\
\text { Approach Based on Units of Meaning }\end{array}$ & $1.35 \mathrm{E}-04$ \\
\hline 口译研究新探 & $\begin{array}{l}\text { An Exploration of Interpreting } \\
\text { Research }\end{array}$ & $7.08 \mathrm{E}-05$ \\
\hline 口译评估 & Interpreting Assessment & $4.26 \mathrm{E}-05$ \\
\hline
\end{tabular}

1 Table 9: The most influential works of Cai Xiaohong 


\section{Table $\mathbf{1 0}$ (on next page)}

The most influential works of Bao Gang 
1

\begin{tabular}{|l|l|c|}
\hline \multicolumn{1}{|c|}{$\begin{array}{c}\text { Cited Work } \\
\text { (original Chinese title) }\end{array}$} & \multicolumn{1}{|c|}{ Englished Title } & PageRank Scores \\
\hline 口译理论概述 & An Overview of Interpreting Theories & 0.00266572 \\
\hline 高校口译训练的方法 & $\begin{array}{l}\text { Preparatory Training for Undergraduate } \\
\text { Interpreting Students }\end{array}$ & $1.51 \mathrm{E}-04$ \\
\hline 口译程序中的思维理解 & $\begin{array}{l}\text { Reasoning and Comprehension in the } \\
\text { Interpreting Process }\end{array}$ & $9.00 \mathrm{E}-05$ \\
\hline 口译程序中的语义问题 & $\begin{array}{l}\text { Semantic Issues in the Interpreting } \\
\text { Process }\end{array}$ & $6.85 \mathrm{E}-05$ \\
\hline 译前准备术语强记的方法论 & $\begin{array}{l}\text { Methods for Memorizing Terms Before } \\
\text { an Assignment }\end{array}$ & $6.81 \mathrm{E}-05$ \\
\hline
\end{tabular}




\section{Table 11 (on next page)}

The most influential works of Liu Heping 
1

\begin{tabular}{|c|c|c|}
\hline $\begin{array}{c}\text { Cited Work } \\
\text { (original Chinese title) }\end{array}$ & Englished Title & PageRank Scores \\
\hline $\begin{array}{l}\text { 对口译教学统一纲要的理论思 } \\
\text { 考 }\end{array}$ & $\begin{array}{l}\text { A Few Thoughts on Standardized } \\
\text { Interpreter Teaching Plans }\end{array}$ & $2.86 \mathrm{E}-04$ \\
\hline 口译理论研究成果与趋势浅析 & Trends in Interpreting Research & 2.70E-04 \\
\hline 翻译的动态研究与口译训练 & $\begin{array}{l}\text { Research in Translator and Interpreter } \\
\text { Training }\end{array}$ & $2.57 \mathrm{E}-04$ \\
\hline $\begin{array}{l}\text { 口译技巧：思维科学与口译推 } \\
\text { 理教学法 }\end{array}$ & $\begin{array}{l}\text { Interpreting Techniques: Scientific } \\
\text { Thinking and Reasoning }\end{array}$ & $1.15 \mathrm{E}-04$ \\
\hline $\begin{array}{l}\text { 口译理论与教学现状研究及展 } \\
\text { 望 }\end{array}$ & $\begin{array}{l}\text { Interpreting Theories and Teaching of } \\
\text { Today and Tomorrow }\end{array}$ & $4.45 \mathrm{E}-05$ \\
\hline
\end{tabular}

Table 11: The most influential works of Liu Heping 


\section{Table 12 (on next page)}

Statistically significant themes in multinomial regression for PageRank 


\begin{tabular}{|l|l|l|l|}
\hline Influence group & Theme group & $\begin{array}{l}\text { Change in likelihood of } \\
\text { belonging to an influence } \\
\text { group (\%) }\end{array}$ & $\begin{array}{l}\text { p- } \\
\text { value }\end{array}$ \\
\hline \multirow{2}{*}{$\begin{array}{l}\text { Medium }(>=20 \text { th, }<85 \text { th } \\
\text { percentile) }\end{array}$} & Cognitive & -0.4 & 0.068 \\
\cline { 2 - 4 } & Socio-Cultural & -0.6 & 0.009 \\
\hline High $(>=85$ th percentile) & Miscellaneous & 0.7 & 0.029 \\
\hline
\end{tabular}

2 Table 12: Statistically significant themes in multinomial regression for PageRank 
Table $\mathbf{1 3}$ (on next page)

Statistically significant themes in multinomial regression for In-Degree 


\begin{tabular}{|c|c|c|c|c|}
\hline \multicolumn{2}{|l|}{ Influence group } & \multirow{2}{*}{$\begin{array}{l}\text { Theme group } \\
\text { Language }\end{array}$} & \multirow{2}{*}{$\begin{array}{l}\text { Change in likelihood of } \\
\text { belonging to an influence } \\
\text { group (\%) }\end{array}$} & \multirow{2}{*}{$\begin{array}{l}\text { p-value } \\
0.011\end{array}$} \\
\hline $\begin{array}{l}\text { Medium } \\
\text { percentile, } \\
\text { percentile })\end{array}$ & $\begin{array}{l}\text { 60th } \\
\text { 95th }\end{array}$ & & & \\
\hline $\operatorname{High}(>=95$ th $\mathrm{p}$ & ntile) & Socio-Cultural & 1.11 & 0.006 \\
\hline
\end{tabular}

1 Table 13: Statistically significant themes in multinomial regression for In-Degree

2

3 
Table 14(on next page)

Statistically significant themes in multinomial regression for Out-Degree 


\begin{tabular}{|l|l|l|l|}
\hline Influence group & $\begin{array}{l}\text { Theme } \\
\text { group }\end{array}$ & $\begin{array}{l}\text { Change in likelihood of } \\
\text { belonging to an influence } \\
\text { group (\%) }\end{array}$ & -value \\
\hline Low (= 0th percentile) & Language & -0.4 & 0.094 \\
\hline $\begin{array}{l}\text { Medium (> 0th percentile, } \\
<80 \text { th percentile) }\end{array}$ & Professional & 0.8 & 0.036 \\
\hline High (>= 80th percentile) & Cognitive & 0.9 & 0.000 \\
\hline
\end{tabular}

1 Table 14: Statistically significant themes in multinomial regression for Out-Degree. 


\section{Table $\mathbf{1 5}$ (on next page)}

F-test for different network influence measures 
1

\begin{tabular}{|l|l|l|l|l|}
\hline & PageRank & In-Degree & Out-Degree & EigenVector Centrality \\
\hline$P$-value & 0.348 & 0.183 & 0.01 & 0.176 \\
\hline
\end{tabular}

2 Table 15: F-test for different network influence measures

3 
Table $\mathbf{1 6}$ (on next page)

Linear regression output of themes on out-degree centrality measure 
1

\begin{tabular}{|l|c|c|c|}
\hline Coefficients & Estimate & Std. Error & $\operatorname{Pr}(>|t|)$ \\
\hline (Intercept) & 8.85850 & 1.17883 & $8.3 \mathrm{e}-14$ \\
\hline No. of papers & $\mathbf{0 . 5 9 4 7 7}$ & $\mathbf{0 . 3 6 0 4 8}$ & $\mathbf{0 . 0 9 9 1}$ \\
\hline Cognitive & $\mathbf{0 . 0 8 3 8 1}$ & $\mathbf{0 . 0 2 6 8 7}$ & $\mathbf{0 . 0 0 1 8}$ \\
\hline Language & $\mathbf{0 . 0 6 4 2 9}$ & $\mathbf{0 . 0 2 6 5 6}$ & $\mathbf{0 . 0 1 5 6}$ \\
\hline Miscellaneous & 0.04645 & 0.03449 & 0.1783 \\
\hline Professional & 0.04377 & 0.04183 & 0.2955 \\
\hline Socio-cultural & -0.00565 & 0.02865 & 0.8436 \\
\hline Multiple R-squared & 0.0078 & & \\
\hline Adjusted R-squared & 0.00501 & & \\
\hline F-statistic: $p$-value & $\mathbf{0 . 0 1 0 3}$ & & \\
\hline
\end{tabular}

2 Table 16: Linear regression output of themes on out-degree centrality measure

3

4 


\section{Table 17 (on next page)}

Gini Coefficients for four network influence measures 
1

\begin{tabular}{|l|l|l|l|l|}
\hline Network Influence & PageRank & In-Degree & Out-Degree & EigenVector \\
\hline Gini Coefficient & 0.28 & 0.83 & 0.81 & 0.84 \\
\hline
\end{tabular}

2 Table 17: Gini Coefficients for four network influence measures

3 
Table $\mathbf{1 8}$ (on next page)

Optimal cutoff quantiles and their measure values 
1

\begin{tabular}{|l|c|c|c|c|}
\hline & $\begin{array}{c}\text { Low Cutoff } \\
\text { (percentile) }\end{array}$ & $\begin{array}{c}\text { Low Cutoff } \\
\text { (Val) }\end{array}$ & $\begin{array}{c}\text { High Cutoff } \\
\text { (percentile) }\end{array}$ & $\begin{array}{c}\text { High Cutoff } \\
\text { (Val) }\end{array}$ \\
\hline PageRank & 20 & 0.0000659 & 85 & 0.0000877 \\
\hline In-Degree & 60 & 2 & 95 & 33 \\
\hline Out-Degree & 60 & 0 & 80 & 24 \\
\hline
\end{tabular}

2 Table 18: Optimal cutoff quantiles and their measure values

3 
Table $\mathbf{1 9}$ (on next page)

PageRank Multinomial Regression 
1

\begin{tabular}{|l|c|c|c|}
\hline Low $(<20$ th percentile) & coef & se & p values \\
\hline Intercept & $\mathbf{- 0 . 2 7 0}$ & $\mathbf{0 . 1 1 0}$ & $\mathbf{0 . 0 1 4}$ \\
\hline No. of papers & 0.000 & 0.031 & 1.000 \\
\hline cognitive & 0.002 & 0.002 & 0.508 \\
\hline language & 0.002 & 0.002 & 0.432 \\
\hline miscellaneous & 0.000 & 0.003 & 1.000 \\
\hline professional & 0.000 & 0.004 & 0.978 \\
\hline socio-cultural & 0.000 & 0.003 & 1.000 \\
\hline Medium $(>=$ 20th, $<$ 85th percentile) & & & \\
\hline Intercept & $\mathbf{0 . 9 8 8}$ & $\mathbf{0 . 0 9 5}$ & $\mathbf{0 . 0 0 0}$ \\
\hline No. of papers & -0.036 & 0.028 & 0.207 \\
\hline cognitive & $\mathbf{- 0 . 0 0 4}$ & $\mathbf{0 . 0 0 2}$ & $\mathbf{0 . 0 6 8}$ \\
\hline language & -0.001 & 0.002 & 0.763 \\
\hline miscellaneous & -0.003 & 0.003 & 0.276 \\
\hline professional & -0.002 & 0.003 & 0.569 \\
\hline socio-cultural & $\mathbf{- 0 . 0 0 6}$ & $\mathbf{0 . 0 0 2}$ & $\mathbf{0 . 0 0 9}$ \\
\hline High ( $>$ 85th percentile) & & & \\
\hline Intercept & $\mathbf{- 0 . 7 1 9}$ & $\mathbf{0 . 1 2 9}$ & $\mathbf{0 . 0 0 0}$ \\
\hline No. of papers & 0.003 & 0.035 & 0.936 \\
\hline cognitive & 0.000 & 0.003 & 1.000 \\
\hline language & 0.000 & 0.003 & 1.000 \\
\hline miscellaneous & $\mathbf{0 . 0 0 7}$ & $\mathbf{0 . 0 0 3}$ & $\mathbf{0 . 0 2 9}$ \\
\hline professional & 0.000 & 0.005 & 1.000 \\
\hline socio-cultural & 0.002 & 0.003 & 0.512 \\
\hline Tab & & & \\
\hline
\end{tabular}

2 Table 19: PageRank Multinomial Regression 


\section{Table $\mathbf{2 0}$ (on next page)}

In-Degree Multinomial Regression 
1

\begin{tabular}{|l|c|c|c|}
\hline Low $(<60$ th percentile) & coef & se & p values \\
\hline Intercept & $\mathbf{1 . 2 3 7}$ & $\mathbf{0 . 0 9 7}$ & $\mathbf{0 . 0 0 0}$ \\
\hline No. of papers & 0.000 & 0.028 & 1.000 \\
\hline cognitive & -0.001 & 0.002 & 0.677 \\
\hline language & 0.000 & 0.002 & 1.000 \\
\hline miscellaneous & -0.003 & 0.003 & 0.309 \\
\hline professional & -0.002 & 0.003 & 0.473 \\
\hline socio-cultural & 0.000 & 0.002 & 1.000 \\
\hline Medium ( $>=60$ th percentile, $<$ 95th percentile) & & & \\
\hline Intercept & $\mathbf{0 . 1 9 4}$ & $\mathbf{0 . 1 0 1}$ & $\mathbf{0 . 0 5 4}$ \\
\hline No. of papers & 0.041 & 0.029 & 0.153 \\
\hline cognitive & 0.000 & 0.002 & 1.000 \\
\hline language & $\mathbf{0 . 0 0 6}$ & $\mathbf{0 . 0 0 2}$ & $\mathbf{0 . 0 1 1}$ \\
\hline miscellaneous & 0.000 & 0.003 & 1.000 \\
\hline professional & 0.001 & 0.004 & 0.799 \\
\hline socio-cultural & -0.001 & 0.003 & 0.613 \\
\hline High $(>=95$ th percentile) & & & \\
\hline Intercept & $\mathbf{- 1 . 4 3 1}$ & $\mathbf{0 . 2 3 8}$ & $\mathbf{0 . 0 0 0}$ \\
\hline No. of papers & -0.100 & 0.100 & 0.318 \\
\hline cognitive & 0.002 & 0.005 & 0.739 \\
\hline language & -0.003 & 0.005 & 0.601 \\
\hline miscellaneous & 0.005 & 0.005 & 0.308 \\
\hline professional & 0.000 & 0.007 & 1.000 \\
\hline socio-cultural & $\mathbf{0 . 0 1 1}$ & $\mathbf{0 . 0 0 4}$ & $\mathbf{0 . 0 0 6}$ \\
\hline
\end{tabular}

2 Table 20: In-Degree Multinomial Regression 


\section{Table $\mathbf{2 1}$ (on next page)}

Out-Degree Multinomial Regression 
1

\begin{tabular}{|l|c|c|c|}
\hline Low (= 0th percentile) & coef & se & p values \\
\hline Intercept & $\mathbf{1 . 0 1 4}$ & $\mathbf{0 . 0 9 6}$ & $\mathbf{0 . 0 0 0}$ \\
\hline No. of papers & 0.000 & 0.029 & 1.000 \\
\hline cognitive & 0.000 & 0.002 & 1.000 \\
\hline language & $\mathbf{- 0 . 0 0 4}$ & $\mathbf{0 . 0 0 2}$ & $\mathbf{0 . 0 9 4}$ \\
\hline miscellaneous & -0.004 & 0.003 & 0.180 \\
\hline professional & 0.000 & 0.003 & 1.000 \\
\hline socio-cultural & -0.002 & 0.002 & 0.372 \\
\hline Medium ( $>$ 0th percentile, $<$ 80th percentile) & & & \\
\hline Intercept & $\mathbf{- 0 . 4 6 9}$ & $\mathbf{0 . 1 3 0}$ & $\mathbf{0 . 0 0 0}$ \\
\hline No. of papers & -0.029 & 0.046 & 0.528 \\
\hline cognitive & -0.001 & 0.003 & 0.837 \\
\hline language & 0.000 & 0.003 & 1.000 \\
\hline miscellaneous & 0.001 & 0.003 & 0.813 \\
\hline professional & $\mathbf{0 . 0 0 8}$ & $\mathbf{0 . 0 0 4}$ & $\mathbf{0 . 0 3 6}$ \\
\hline Socio-cultural & 0.001 & 0.003 & 0.647 \\
\hline High $(>=80 t h$ percentile) & & & \\
\hline Intercept & $\mathbf{- 0 . 5 4 5}$ & $\mathbf{0 . 1 1 7}$ & $\mathbf{0 . 0 0 0}$ \\
\hline No. of papers & $\mathbf{0 . 0 7 5}$ & $\mathbf{0 . 0 3 1}$ & $\mathbf{0 . 0 1 4}$ \\
\hline cognitive & $\mathbf{0 . 0 0 9}$ & $\mathbf{0 . 0 0 2}$ & $\mathbf{0 . 0 0 0}$ \\
\hline language & 0.002 & 0.003 & 0.339 \\
\hline miscellaneous & 0.000 & 0.003 & 1.000 \\
\hline professional & -0.001 & 0.005 & 0.900 \\
\hline socio-cultural & 0.000 & 0.003 & 1.000 \\
\hline Tale 1 Out- & & \\
\hline
\end{tabular}

2 Table 21: Out-Degree Multinomial Regression 


\title{
Table 22 (on next page)
}

\author{
PageRank Keyword Profile Regression
}




\begin{tabular}{|l|l|l|l|}
\hline Keyword & $\begin{array}{l}\text { Low/Mid/High } \\
\text { Group }\end{array}$ & $\begin{array}{l}\text { Positive/Negative } \\
\text { Association }\end{array}$ & Theme Group \\
\hline Attention & Low & Positive & Cognitive \\
\hline Nominalization & Low & Positive & Language \\
\hline Extralinguistic Information & Medium & Negative & Socio-Cultural \\
\hline Interpreters Roles & Medium & Negative & Socio-Cultural \\
\hline Interpreting Process & Medium & Negative & Cognitive \\
\hline Schemata & Medium & Negative & Cognitive \\
\hline Coping Tactics & Medium & Positive & Training \\
\hline Theory & High & Positive & Miscellaneous \\
\hline Case Studies & Low & Negative & Training \\
\hline Domestication & Low & Negative & Language \\
\hline Faithfulness & Low & Negative & Language \\
\hline Fidelity & Low & Negative & Training \\
\hline Cultural Awareness & Medium & Negative & Language \\
\hline Interpreting Process & Medium & Negative & Training \\
\hline Content Validity & Medium & Positive & Language \\
\hline Faithfulness & Medium & Positive & Professional \\
\hline Interpreting Styles & Medium & Positive & Language \\
\hline Logic & Medium & Positive & Training \\
\hline Non Standard Expressions & Medium & Positive & Miscellaneous \\
\hline Rights & Medium & Positive & Socio-Cultural \\
\hline Tem8 & Medium & Positive & Language \\
\hline Thematic Progression & Medium & Positive & Language \\
\hline Certification & Positive & Socio-Cultural \\
\hline
\end{tabular}




\begin{tabular}{|l|l|l|l|}
\hline Nonlinguistic Context & Medium & Positive & Professional \\
\hline Rhetoric Fuzziness & Medium & Positive & Language \\
\hline Textual Coherence & Medium & Positive & Language \\
\hline Copyright & Medium & Positive & Cognitive \\
\hline Self Evaluation & Medium & Positive & Training \\
\hline Chinese Classics & Medium & Positive & Language \\
\hline
\end{tabular}

2 Table 22: PageRank Keyword Profile Regression 
Table $\mathbf{2 3}$ (on next page)

In-Degree Keyword Profile Regression 


\begin{tabular}{|c|c|c|c|}
\hline Keyword & $\begin{array}{l}\text { Low/Mid/High } \\
\text { Group }\end{array}$ & $\begin{array}{l}\text { Positive/Negative } \\
\text { Association }\end{array}$ & Theme Group \\
\hline Case Studies & Low & Negative & Training \\
\hline Domestication & Low & Negative & Language \\
\hline Faithfulness & Low & Negative & Language \\
\hline Fidelity & Low & Negative & Training \\
\hline $\begin{array}{l}\text { Cultural } \\
\text { Awareness }\end{array}$ & Medium & Negative & Cognitive \\
\hline $\begin{array}{l}\text { Interpreting } \\
\text { Process }\end{array}$ & Medium & Negative & Training \\
\hline Content Validity & Medium & Positive & Language \\
\hline Faithfulness & Medium & Positive & Professional \\
\hline $\begin{array}{l}\text { Interpreting } \\
\text { Styles }\end{array}$ & Medium & Positive & Language \\
\hline Logic & Medium & Positive & Training \\
\hline $\begin{array}{l}\text { Non-Standard } \\
\text { Expressions }\end{array}$ & Medium & Positive & Miscellaneous \\
\hline Rights & Medium & Positive & Socio-Cultural \\
\hline Tem8 & Medium & Positive & Professional \\
\hline $\begin{array}{l}\text { Thematic } \\
\text { Progression }\end{array}$ & Medium & Positive & Language \\
\hline Certification & Medium & Positive & Socio-Cultural \\
\hline $\begin{array}{l}\text { Non-linguistic } \\
\text { Context }\end{array}$ & Medium & Positive & Professional \\
\hline $\begin{array}{l}\text { Rhetoric } \\
\text { Fuzziness }\end{array}$ & Medium & Positive & Language \\
\hline $\begin{array}{l}\text { Textual } \\
\text { Coherence }\end{array}$ & Medium & Positive & Language \\
\hline Self-evaluation & Medium & Positive & Training \\
\hline
\end{tabular}




\begin{tabular}{|l|l|l|l|}
\hline Chinese Classics & Medium & Positive & Language \\
\hline
\end{tabular}

2 Table 23: In-Degree Keyword Profile Regression 


\section{Table 24(on next page)}

Out-Degree Keyword Profile Regression 
1

\begin{tabular}{|l|l|l|l|}
\hline Keyword & $\begin{array}{l}\text { Low/Mid/High } \\
\text { Group }\end{array}$ & $\begin{array}{l}\text { Positive/Negative } \\
\text { Association }\end{array}$ & Theme Group \\
\hline Attention & Low & Negative & Cognitive \\
\hline Sensory Memory & High & Positive & Cognitive \\
\hline Trade Association & High & Positive & Professional \\
\hline
\end{tabular}

2 Table 24: Out-Degree Keyword Profile Regression

3 